\author{
DAniah TAhir. ID , Ingemar KaJ (D) (Uppsala) \\ KRZYSZTOF BARTOSZEK iD * (Linköping) \\ Marta Majchrzak (iD), Pawel Parniewsk] iD * (Eódź) \\ SEbastian SAKOWskl (E) (Eódź)
}

\title{
Using multitype branching models to analyze bacterial pathogenicity
}

\begin{abstract}
We apply multitype continuous time Markov branching models to study pathogenicity in E. coli, a bacterium belonging to the genus Escherichia. First, we examine briefly the properties of multitype branching processes and we also survey some fundamental limit theorems regarding the behavior of such models under various conditions. These theorems are then applied to discrete, state dependent models in order to analyze pathogenicity in a published clinical data set consisting of 251 strains of $E$. coli. We use well established methods, incorporating maximum likelihood techniques, to estimate speciation rates as well as the rates of transition between different states of the models. From the analysis, we not only derive new results, but we also verify some preexisting notions about virulent behavior in bacterial strains.
\end{abstract}

2010 Mathematics Subject Classification: Primary: 60B12; Secondary: 60J85.

Key words and phrases: Markov models, branching processes, limit theorems, virulence factors, E. coli strains.

1. Introduction In this paper, we explore the possibility of utilizing the theory of branching processes into analyzing pathogenicity in bacterial strains. For that purpose, we first review fundamental properties of multitype, continuous time Markov branching processes as well as their behavior in the long time limit. Then, we apply multitype branching models to examine virulence in E. coli strains, and perform an in depth analysis on the limits of proportions of E. coli in different states of the models. The strains used in this study were isolated from human hosts, and obtained from a previously published data set (by Bartoszek et al. [4]) of pathogenic and nonpathogenic E. coli bacteria.

In recent years, considerable research has been conducted regarding the use of branching processes to explore various biological phenomena. A twotype Markov branching model, coined as the 'binary state speciation and extinction' (BiSSE) model, was proposed by Maddison et al. [12] to assess

* KB was supported by Vetenskapsrådets grant no. 2017-04951.

* MM and PP were partially supported by IMB PAS. 
the impact of binary characters on rates of diversification - the difference between speciation and extinction rates. The parameters of the BiSSE model describe speciation and extinction in the two types, as well as the transitions that take place from one type to the other. This model was recently used by Bartoszek et al. [4] in order to estimate parameters from genetic data of E. coli populations, and predict pathogenicity of various virulence factors (VFs) - agents that enable bacteria to replicate and spread within the host by damaging and eluding its defences [6]. Bartoszek et al. used a modified version of the BiSSE model, in which the extinction rates were assumed to be zero. Another Markov branching model, named as the 'multistate speciation and extinction' (MuSSE) model, was later introduced by FitzJohn [7] as an extension of the BiSSE model to binary traits with more than two states.

In this paper, we apply the MuSSE model with zero extinction rates to estimate state dependent speciation and transition rates for a real, known collection of pathogenic and nonpathogenic strains of E. coli bacteria (obtained from [4]) that reside in the human digestive and urinary tracts. The bacterial strains are subdivided into four categories depending on whether or not they carry a VF in the gut and bladder of the human host. Lately, a number of researchers have successfully used the discrete state MuSSE model for estimation of various parameters. For instance, Sachs et al. (2013) used the MuSSE model to estimate trait-dependent diversification and transition rates amongst states consisting of free-living, mutualistic, parasitic, and dual lifestyle bacteria in the Proteobacteria phylum. Using this model, they inferred that proteobacterial mutualist lineages arise from free-living and parasitic ancestors, but rarely transition back to a parasitic or free-living status. Pirie et al. [14] implemented the MuSSE framework to compare diversification rates of 800 species in the plant genus Erica, endemic to five geographical regions-Palearctic, Tropical African, Madagascan, Drakensberg and Cape. Arbuckle et al. [1] used the BiSSE and MuSSE models to show that speciation and extinction rates vary across defensive traits in amphibians.

With this study, we ask the following questions:

(a) Is it possible to utilize state dependent branching processes to analyze pathogenic behavior in bacteria?

(b) How do maximum likelihood methods behave when estimating parameters (such as speciation rates and transition rates between states) of multitype models?

(c) Based only on a finite sample, do the estimated parameters provide reasonable information on the almost sure limits of the proportion of bacterial strains, and if so, can they be used further to obtain plausible confidence regions for these limits?

To answer these questions, we proceed by first giving a concise survey of $n$-type branching processes. More specifically, we recall fundamental theorems 
regarding the long time behavior of branching models. These limit theorems are obtained from earlier works of Athreya and Ney [2] and Janson [8]. In order to thoroughly explain the mathematical background, which is pertinent to understanding the forthcoming biological application, we introduce some technical notation as well. For multitype branching models, a matrix known as the mean offspring matrix - whose entries consist of the net growth and transition rates of the process - is of central importance; the limit behavior of the process can be completely characterized by this matrix through its eigenvalues and corresponding eigenvectors. In the coming sections, we evaluate the mean offspring matrix for various sub-models of the multitype branching process, and present interesting results associated with the largest eigenvalue of these matrices. After reviewing the general characteristics of multitype branching processes, we apply 4-type branching sub-models to a known clinical data set of virulent and nonvirulent $E$. coli strains, and, with the help of the MuSSE model, obtain rates of speciation and transition among the 4 states of the models. Using the aforementioned limit theorems, we also perform an in depth analysis on the limits of proportions of E. coli strains in different states. Finally, we compare the results obtained from various models and draw some useful inferences regarding pathogenic behavior in virulent bacteria.

2. General multitype branching processes We consider an $n$-type continuous time Markov branching process $\boldsymbol{X}(t)$, given by the column vector $\boldsymbol{X}(t)=\left(X_{1}(t), \ldots, X_{n}(t)\right)^{\prime}, t \geq 0$, where each $X_{i}(t), i=1, \ldots, n$, represents the number of type- $i$ particles at time $t$. The lifetime of each type is assumed to be exponentially distributed with intensity $a_{i}, i=1, \ldots, n$. We introduce a vector $\boldsymbol{a}$ whose components comprise of $a_{i}$, i.e., $\boldsymbol{a}=\left(a_{1}, \ldots, a_{n}\right)$. With each type $i$, we also associate $\boldsymbol{j}=\left(j_{1}, \ldots, j_{n}\right)$, a vector of nonnegative integer coordinates. Then, the offspring distribution of the $n$ types is specified by the coordinates of $\boldsymbol{p}(\boldsymbol{j})$, where

$$
\boldsymbol{p}(\boldsymbol{j})=\left(p^{(1)}(\boldsymbol{j}), \ldots, p^{(n)}(\boldsymbol{j})\right)
$$

and $\sum_{\boldsymbol{j}} p^{(i)}(\boldsymbol{j})=1$, for all $i=1, \ldots, n$. Here, $p^{(i)}(\boldsymbol{j})=p^{(i)}\left(j_{1}, \ldots, j_{n}\right)$ gives the probability that a type- $i$ particle creates $j_{1}$ type- 1 offspring, $j_{2}$ type-2 offspring, $\ldots, j_{n}$ type- $n$ offspring [3]. Letting $s=\left(s_{1}, \ldots, s_{n}\right)$, the generating function is recognized as $\boldsymbol{f}(\boldsymbol{s})=\left(f^{(1)}(\boldsymbol{s}), \ldots, f^{(n)}(\boldsymbol{s})\right)$, where, for each $i=$ $1, \ldots, n$,

$$
f^{(i)}(\boldsymbol{s})=\sum_{\boldsymbol{j}} p^{(i)}(\boldsymbol{j}) \boldsymbol{s}^{\boldsymbol{j}}=\sum_{j_{1}, \ldots, j_{n} \geq 0} p^{(i)}\left(j_{1}, \ldots, j_{n}\right) s_{1}^{j_{1}} \cdot \ldots \cdot s_{n}^{j_{n}}
$$

determines the distribution of the number of various types of offspring produced by a type- $i$ particle. Further, we consider the matrix $\mathcal{A}=\left\{a_{i k}: i, k=\right.$ $1, \ldots, n\}$, where 


$$
a_{i k}=a_{i}\left(\left.\frac{\partial f^{(i)}(s)}{\partial s_{k}}\right|_{s=(1, \ldots, 1)}-\delta_{i k}\right) \quad \text { and } \quad \delta_{i k}=\left\{\begin{array}{llr}
1 & \text { if } \quad i=k \\
0 & \text { otherwise }
\end{array}\right.
$$

The mean matrix of the branching process is given by

$$
M(t)=e^{\mathcal{A} t}=\left\{m_{i k}(t): i, k=1, \ldots, n\right\},
$$

with $m_{i k}(t)=\mathbb{E}\left[X_{k}(t) \mid X_{i}(t)=1\right]$ [3]. Following [8], we identify the mean offspring matrix $\boldsymbol{A}$ as

$$
\boldsymbol{A}=\mathcal{A}^{T},
$$

where $T$ in the superscript denotes the matrix transpose. We let $\gamma$ be the largest positive eigenvalue of $\boldsymbol{A}$, and, let $\boldsymbol{u}$ and $\boldsymbol{v}$ be the left and right normalized column eigenvectors, respectively, of $\boldsymbol{A}$, corresponding to $\gamma$. Thus, $\boldsymbol{u}^{\prime} \boldsymbol{A}=\gamma \boldsymbol{u}^{\prime}$ and $\boldsymbol{A} \boldsymbol{v}=\gamma \boldsymbol{v}$.

We now recall some limit results for multitype branching processes that will be used in later sections for the analysis of branching models. These results, numbered 1, 2 and 3 here, are stated formally in Appendix A of the paper as Theorems A.3, A.4 and A.5, respectively.

1. The fundamental limit result for multitype branching processes signifies the existence of a nonnegative random variable $W$ such that $e^{-\gamma t} \boldsymbol{X}(t) \stackrel{\text { a.s. }}{\longrightarrow} W \boldsymbol{v}$ as $t \rightarrow \infty$ [2]. This implies that the number of particles increases to infinity at a speed $e^{\gamma t}$, with the distribution of the types specified by a random multiple of the right eigenvector.

2. For a real nonnegative number $N$, let $T_{N}$ be the first time when the total size of the branching process reaches a given level $N>0$. Then, letting $C$ be the sum of the components of $\boldsymbol{v}$, and under additional assumptions (see Appendix A), the asymptotic distribution of types at $T_{N}$ is given by the right normalized eigenvector $\boldsymbol{v}$ in the sense that $\boldsymbol{X}\left(T_{N}\right) / N \stackrel{\text { a.s. }}{\longrightarrow} \boldsymbol{v} / C$ as $N \rightarrow \infty$ [8].

3. Under further assumptions on the size of the eigenvalues of the offspring matrix $\boldsymbol{A}$, as $N \rightarrow \infty$, the centered and normalized type distribution has a Gaussian limit distribution in the sense that $\sqrt{N}\left(\boldsymbol{X}\left(T_{N}\right) / N-\boldsymbol{v} / C\right)$ $\stackrel{\mathcal{D}}{\longrightarrow} \mathcal{N}\left(0, \boldsymbol{\Sigma}_{\boldsymbol{b}}\right)$, where $\mathcal{N}\left(0, \boldsymbol{\Sigma}_{\boldsymbol{b}}\right)$ is a multivariate normal distribution and $\boldsymbol{\Sigma}_{\boldsymbol{b}}$ is the covariance matrix stated explicitly in Appendix A [8].

3. A 4-type branching model Consider a 4-type, continuous time Markov branching process, $\boldsymbol{X}(t)=\left(X_{1}(t), \ldots, X_{4}(t)\right)^{\prime}, t \geq 0$, where each $X_{i}(t), i=1, \ldots, 4$, gives the number of type $-i$ particles at time $t$. Let $\lambda_{i}$ represent the speciation rate of type- $i$ particles, $i=1, \ldots, 4$. Further, $q_{12}$ and $q_{21}$ are the rates of transition from type- $1 \rightarrow 2$ and type- $2 \rightarrow 1$, respectively, and similarly, $q_{34}$ and $q_{43}$ the transition rates from type- $3 \rightarrow 4$ and type$4 \rightarrow 3$, respectively. Finally, $m_{32}$ is assumed to be the transition rate from type -3 to type -2 . The branching rates of $\boldsymbol{X}(t)$ are thus given as 


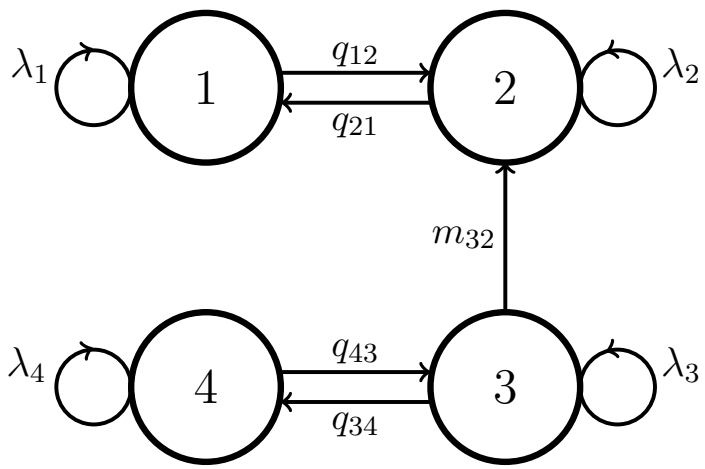

Figure 1: Diagrammatic representation of the speciation and transition parameters in the 4-type branching model $\boldsymbol{X}(t)$.

$$
\left(x_{1}, x_{2}, x_{3}, x_{4}\right) \mapsto\left\{\begin{array}{cc}
\left(x_{1}+1, x_{2}, x_{3}, x_{4}\right) & \lambda_{1} x_{1} \\
\left(x_{1}, x_{2}+1, x_{3}, x_{4}\right) & \lambda_{2} x_{2} \\
\left(x_{1}, x_{2}, x_{3}+1, x_{4}\right) & \lambda_{3} x_{3} \\
\left(x_{1}, x_{2}, x_{3}, x_{4}+1\right) & \lambda_{4} x_{4} \\
\left(x_{1}-1, x_{2}+1, x_{3}, x_{4}\right) & q_{12} x_{1} \\
\left(x_{1}+1, x_{2}-1, x_{3}, x_{4}\right) & q_{21} x_{2} \\
\left(x_{1}, x_{2}, x_{3}-1, x_{4}+1\right) & q_{34} x_{3} \\
\left(x_{1}, x_{2}, x_{3}+1, x_{4}-1\right) & q_{43} x_{4} \\
\left(x_{1}, x_{2}+1, x_{3}-1, x_{4}\right) & m_{32} x_{3},
\end{array}\right.
$$

where the initial state $\boldsymbol{X}(0)$ can be either $(0,0,1,0)^{\prime}$ or $(0,0,0,1)^{\prime}$, since type3 and type -4 are the dominating types (see Def. A.1). Figure 1 summarizes the parameters used in this branching process. The motivation behind choosing this particular model is that the results derived here will be applied in Section 4 to obtain results and draw inferences from a real data set of $E$. coli bacterial strains. Note that a 4-type branching model would generally consist of 20 parameters: 4 speciation parameters, 4 extinction parameters and 12 parameters representing transition rates between states. Here we have assumed nonextinction, and we have also set 7 (out of 12) transition parameters to zero.

In order to identify the offspring matrix $\boldsymbol{A}$ for this process, we recall notation from Section 2, and let $\boldsymbol{a}=\left(a_{1}, \ldots, a_{4}\right)$, where

$$
a_{1}=\lambda_{1}+q_{12}, \quad a_{2}=\lambda_{2}+q_{21}, \quad a_{3}=\lambda_{3}+q_{34}+m_{32}, \quad a_{4}=\lambda_{4}+q_{43} .
$$

We recognize the offspring distribution of the four states as

$$
\begin{gathered}
p^{(1)}(2,0,0,0)=\lambda_{1} / a_{1}, \quad p^{(1)}(0,1,0,0)=q_{12} / a_{1}, \\
p^{(2)}(0,2,0,0)=\lambda_{2} / a_{2}, \quad p^{(2)}(1,0,0,0)=q_{21} / a_{2}, \\
p^{(3)}(0,0,2,0)=\lambda_{3} / a_{3}, \quad p^{(3)}(0,0,0,1)=q_{34} / a_{3}, \quad p^{(3)}(0,1,0,0)=m_{32} / a_{3},
\end{gathered}
$$




$$
p^{(4)}(0,0,0,2)=\lambda_{4} / a_{4}, \quad p^{(4)}(0,0,1,0)=q_{43} / a_{4} .
$$

Using the above information, we are now able to find the generating functions:

$$
\begin{gathered}
f^{(1)}\left(s_{1}, s_{2}, s_{3}, s_{4}\right)=\left(\lambda_{1} s_{1}^{2}+q_{12} s_{2}\right) / a_{1}, \\
f^{(2)}\left(s_{1}, s_{2}, s_{3}, s_{4}\right)=\left(\lambda_{2} s_{2}^{2}+q_{21} s_{1}\right) / a_{2}, \\
f^{(3)}\left(s_{1}, s_{2}, s_{3}, s_{4}\right)=\left(\lambda_{3} s_{3}^{2}+q_{34} s_{4}+m_{32} s_{2}\right) / a_{3}, \\
f^{(4)}\left(s_{1}, s_{2}, s_{3}, s_{4}\right)=\left(\lambda_{4} s_{4}^{2}+q_{43} s_{3}\right) / a_{4} .
\end{gathered}
$$

The mean offspring matrix $\boldsymbol{A}$ for this process is defined as

$$
\boldsymbol{A}=\left(\begin{array}{cccc}
\delta_{1} & q_{21} & 0 & 0 \\
q_{12} & \delta_{2} & m_{32} & 0 \\
0 & 0 & \delta_{3} & q_{43} \\
0 & 0 & q_{34} & \delta_{4}
\end{array}\right)
$$

where $\delta_{1}=\lambda_{1}-q_{12}, \delta_{2}=\lambda_{2}-q_{21}, \delta_{3}=\lambda_{3}-m_{32}-q_{34}$ and $\delta_{4}=\lambda_{4}-q_{43}$. Letting

$$
\begin{gathered}
H_{1}=\delta_{1}+\delta_{2}, \quad H_{2}=\delta_{3}+\delta_{4}, \\
S_{1}=\sqrt{\left(\delta_{1}-\delta_{2}\right)^{2}+4 q_{12} q_{21}}, \quad S_{2}=\sqrt{\left(\delta_{3}-\delta_{4}\right)^{2}+4 q_{34} q_{43}},
\end{gathered}
$$

the eigenvalues of $\boldsymbol{A}$, obtained using Maple 18.00 [13], are

$$
\gamma_{1}=\frac{1}{2}\left(H_{1}+S_{1}\right), \quad \gamma_{2}=\frac{1}{2}\left(H_{1}-S_{1}\right), \quad \gamma_{3}=\frac{1}{2}\left(H_{2}+S_{2}\right), \quad \gamma_{4}=\frac{1}{2}\left(H_{2}-S_{2}\right) .
$$

We have

$\gamma_{1} \geq \max \left\{\delta_{1}, \delta_{2}\right\} \geq \min \left\{\delta_{1}, \delta_{2}\right\} \geq \gamma_{2}, \quad \gamma_{3} \geq \max \left\{\delta_{3}, \delta_{4}\right\} \geq \min \left\{\delta_{3}, \delta_{4}\right\} \geq \gamma_{4}$

It can be seen that $\gamma_{1} \geq 0$ and $\gamma_{3} \geq 0$ for any set of parameters. Also, $\gamma_{1}>0$ if at least one of $\delta_{1}$ and $\delta_{2}$ is strictly positive, or if both are negative, then one is strictly negative, and a similar result holds for $\gamma_{3}$. Further, we also require the eigenvectors of $\boldsymbol{A}$ to be used later in the application of limit theorems A.3-A.5. Thus, using again Maple 18.00 [13], the left column eigenvectors of $\boldsymbol{A}$, after substantial manual simplification, are found as

$$
\begin{gathered}
\boldsymbol{u}_{1}=\left(\frac{\frac{1}{2}\left(G_{1}+S_{1}\right)\left(\gamma_{1} G_{3}+G_{4}\right)}{q_{21} m_{32} q_{43}}, \frac{\gamma_{1} G_{3}+G_{4}}{q_{43} m_{32}}, \frac{\gamma_{1}-\lambda_{4}+q_{43}}{q_{43}}, 1\right)^{\prime}, \\
\boldsymbol{u}_{\mathbf{2}}=\left(\frac{-\frac{1}{2}\left(-G_{1}+S_{1}\right)\left(\gamma_{2} G_{3}+G_{4}\right)}{q_{21} m_{32} q_{43}}, \frac{\gamma_{2} G_{3}+G_{4}}{q_{43} m_{32}}, \frac{\gamma_{2}-\lambda_{4}+q_{43}}{q_{43}}, 1\right)^{\prime}, \\
\boldsymbol{u}_{\mathbf{3}}=\left(0,0,-\frac{1}{2 q_{43}}\left(G_{2}-S_{2}\right), 1\right)^{\prime}, \quad \boldsymbol{u}_{4}=\left(0,0,-\frac{1}{2 q_{43}}\left(G_{2}+S_{2}\right), 1\right)^{\prime},
\end{gathered}
$$


and similarly, the right column eigenvectors are computed as

$$
\begin{gathered}
\boldsymbol{v}_{\mathbf{1}}=\left(1,-\frac{1}{2 q_{21}}\left(G_{1}-S_{1}\right), 0,0\right)^{\prime}, \quad \boldsymbol{v}_{\mathbf{2}}=\left(1,-\frac{1}{2 q_{21}}\left(G_{1}+S_{1}\right), 0,0\right)^{\prime} \\
\boldsymbol{v}_{\mathbf{3}}=\left(1, \frac{\gamma_{3}-\lambda_{1}+q_{12}}{q_{21}}, \frac{\gamma_{3} G_{3}+G_{4}}{-q_{21} m_{32}}, \frac{\frac{1}{2}\left(G_{2}+S_{2}\right)\left(\gamma_{3} G_{3}+G_{4}\right)}{-q_{21} m_{32} q_{43}}\right)^{\prime} \\
\boldsymbol{v}_{\mathbf{4}}=\left(1, \frac{\gamma_{4}-\lambda_{1}+q_{12}}{q_{21}}, \frac{\gamma_{4} G_{3}+G_{4}}{-q_{21} m_{32}}, \frac{\frac{1}{2}\left(G_{2}-S_{2}\right)\left(\gamma_{4} G_{3}+G_{4}\right)}{-q_{21} m_{32} q_{43}}\right)^{\prime}
\end{gathered}
$$

where $G_{1}=\delta_{1}-\delta_{2}, G_{2}=\delta_{4}-\delta_{3}, G_{3}=H_{1}-H_{2}$, and $G_{4}=\delta_{3} \delta_{4}-\delta_{1} \delta_{2}-$ $q_{43}\left(m_{32}+q_{34}\right)+q_{12} q_{21}+q_{43} m_{32}$. It should be noted that for certain parameter settings, where one of $q_{21}, q_{43}$ or $m_{32}$ vanish, the above expressions do not apply. Instead, alternate formulae have to be found on a case by case basis.

Now let $\gamma=\max \left\{\gamma_{1}, \gamma_{3}\right\}$ be the largest positive eigenvalue, and let $\boldsymbol{v}=$ $\left(\nu_{1}, \nu_{2}, \nu_{3}, \nu_{4}\right)^{\prime}$ be the normalized form of the corresponding right eigenvector. Then, from Thm. A.3 (and as described in limit result 1), there exists a nonnegative random variable $W$, such that as $t \rightarrow \infty$,

$$
\left(X_{1}(t) e^{-\gamma t}, \ldots, X_{4}(t) e^{-\gamma t}\right)^{\prime} \stackrel{a . s}{\longrightarrow}\left(\nu_{1} W, \ldots, \nu_{4} W\right)^{\prime} .
$$

Moreover, let $\nu_{1}+\nu_{2}+\nu_{3}+\nu_{4}=C>0$ and recall that $T_{N}$ is the first time when the total number of species reaches a level $N$. Using limit result 2 (or Thm. A.4), we have that as $N \rightarrow \infty$,

$$
\frac{\boldsymbol{X}\left(T_{N}\right)}{N}=\frac{\left(X_{1}\left(T_{N}\right), \ldots, X_{4}\left(T_{N}\right)\right)^{\prime}}{N} \stackrel{a . s}{\longrightarrow} \frac{\left(\nu_{1}, \ldots, \nu_{4}\right)^{\prime}}{C} .
$$

In order to apply the limit result 3 (see also Thm. A.5 in Appendix A), we need more information on the variance characteristics of the branching process. For that purpose, consider first the column vectors, $\xi_{i}, i=1, \ldots, 4$, given as

$$
\begin{gathered}
\xi_{1}=\left\{\begin{array}{ccc}
(1,0,0,0)^{\prime} & \text { with probability } & \lambda_{1} / a_{1} \\
(-1,1,0,0)^{\prime} & \text { " }^{\prime} & q_{12} / a_{1}
\end{array},\right. \\
\xi_{2}=\left\{\begin{array}{ccc}
(0,1,0,0)^{\prime} & \text { with probability } & \lambda_{2} / a_{2} \\
(1,-1,0,0)^{\prime} & \text {-" }_{21} / a_{2}
\end{array},\right. \\
\xi_{3}=\left\{\begin{array}{ccc}
(0,0,1,0)^{\prime} & \text { with probability } & \lambda_{3} / a_{3} \\
(0,0,-1,1)^{\prime} & -"- & q_{34} / a_{3} \\
(0,1,-1,0)^{\prime} & -"- & m_{32} / a_{3}
\end{array},\right. \\
\xi_{4}=\left\{\begin{array}{ccc}
(0,0,0,1)^{\prime} & \text { with probability } & \lambda_{4} / a_{4} \\
(0,0,1,-1)^{\prime} & - \text {-" } & q_{43} / a_{4}
\end{array}\right.
\end{gathered}
$$


Next, define a matrix $\boldsymbol{B}$ as

$$
\boldsymbol{B}=\sum_{i=1}^{4} \nu_{i} a_{i} \mathbb{E}\left(\xi_{i} \xi_{i}^{\prime}\right)=\left(\begin{array}{cccc}
b_{1} & -b_{2} & 0 & 0 \\
-b_{2} & b_{3} & -b_{4} & 0 \\
0 & -b_{4} & b_{5} & -b_{6} \\
0 & 0 & -b_{6} & b_{7}
\end{array}\right) \text {, }
$$

where $b_{1}=a_{1} \nu_{1}+q_{21} \nu_{2}, b_{2}=q_{12} \nu_{1}+q_{21} \nu_{2}, b_{3}=a_{2} \nu_{2}+q_{12} \nu_{1}+m_{32} \nu_{3}$, $b_{4}=m_{32} \nu_{3}, b_{5}=a_{3} \nu_{3}+q_{43} \nu_{4}, b_{6}=q_{34} \nu_{3}+q_{43} \nu_{4}$ and $b_{7}=a_{4} \nu_{4}+q_{34} \nu_{3}$. Now, since $\boldsymbol{A}$ is a $4 \times 4$ matrix with 4 distinct eigenvalues, it is diagonalizable. Hence, using Eq. (7) from Appendix A, the matrix $\boldsymbol{\Sigma}_{\boldsymbol{I}}$ is specified by

$$
\boldsymbol{\Sigma}_{\boldsymbol{I}}=\sum_{j: \gamma_{j}<\frac{\gamma}{2}} \sum_{k: \gamma_{k}<\frac{\gamma}{2}} \frac{\hat{u}_{j}^{\prime} \boldsymbol{B} \hat{u}_{k}}{\gamma-\gamma_{j}-\gamma_{k}} \hat{v}_{j} \hat{v}_{k}^{\prime}
$$

where column vectors $\hat{u}_{i}$ and $\hat{v}_{i}$ are determined as

$$
\hat{u}_{i}=\frac{\boldsymbol{u}_{\boldsymbol{i}}}{\boldsymbol{u}_{\boldsymbol{i}} \cdot \boldsymbol{v}_{\boldsymbol{i}}} \text { and } \hat{v}_{i}=\boldsymbol{v}_{\boldsymbol{i}}, \quad i=1, \ldots, 4 .
$$

Finally using Eq. (6), the covariance matrix $\boldsymbol{\Sigma}_{\boldsymbol{b}}$ is given by

$$
\boldsymbol{\Sigma}_{b}=\frac{1}{C^{3}} M_{1} \boldsymbol{\Sigma}_{I} \boldsymbol{M}_{2}
$$

where $\quad \boldsymbol{M}_{\mathbf{1}}=\left(\begin{array}{cccc}\nu_{1}-C & \nu_{1} & \nu_{1} & \nu_{1} \\ \nu_{2} & \nu_{2}-C & \nu_{2} & \nu_{2} \\ \nu_{3} & \nu_{3} & \nu_{3}-C & \nu_{3} \\ \nu_{4} & \nu_{4} & \nu_{4} & \nu_{4}-C\end{array}\right) \quad$ and $\quad \boldsymbol{M}_{\mathbf{2}}=\boldsymbol{M}_{\mathbf{1}}^{T}$.

Under the condition that $\gamma$ is greater than two times the second largest eigenvalue, and using the central limit theorem A.5, we have that

$$
\sqrt{N}\left(\frac{\left(X_{1}\left(T_{N}\right), \ldots, X_{4}\left(T_{N}\right)\right)^{\prime}}{N}-\frac{\left(\nu_{1}, \ldots, \nu_{4}\right)^{\prime}}{C}\right) \stackrel{\mathcal{D}}{\longrightarrow} \mathcal{N}\left(0, \boldsymbol{\Sigma}_{\boldsymbol{b}}\right),
$$

as $N \rightarrow \infty$.

In the following section, we apply the above 4-type model to a clinical data set of 251 bacterial strains, and obtain insightful results concerning pathogenicity in E. coli bacteria. We will take $N=251$, so that $T_{N}$ is the first time when the total size of the branching process reaches 251 , and hence we can obtain the proportion of strains in various states of the model.

\section{Application of the branching model to $E$. coli strains data} From [4], we obtain an E. coli data set of $N=251$ strains, which forms the tips of a given phylogenetic tree (see Fig. 1 and Fig. 3 in [4]). The tree is fixed and describes the genealogical structure of the strains. The tree tips 
are grouped into 4 categories: pathogenic and nonpathogenic E. coli found in the human gastrointestinal tract, and, pathogenic and nonpathogenic E. coli found in the human urinary tract. Whether a bacterial strain is pathogenic or not in any environment, depends on whether or not it is positive for carrying a certain VF (such as toxins, invasins, hemolysins, etc.). Hence, the strains are divided into the following 4 states

\begin{tabular}{|c|c|c|c|}
1 & 2 & 3 & 4 \\
\hline$U_{0}$ & $U_{1}$ & $K_{1}$ & $K_{0}$
\end{tabular}

where

$U_{0}$ : negative for $\mathrm{VF}$ in the urinary tract,

$U_{1}$ : positive for $\mathrm{VF}$ in the urinary tract,

$K_{1}$ : positive for $\mathrm{VF}$ in the digestive tract,

$K_{0}$ : negative for $\mathrm{VF}$ in the digestive tract.

\begin{tabular}{lcccc} 
Virulence Factor (VF) & $N_{1}$ & $N_{2}$ & $N_{3}$ & $N_{4}$ \\
\hline astA - heat-stable enterotoxin 1 & 108 & 20 & 7 & 116 \\
\hline cnf1 - cytotoxic necrotizing factor 1 & 90 & 38 & 3 & 120 \\
\hline fimG - fimbrial protein & 13 & 115 & 120 & 3 \\
\hline fyuA - pesticin receptor protein & 50 & 78 & 74 & 49 \\
\hline hly1 - alpha hemolysin & 88 & 40 & 5 & 118 \\
\hline iroN - IroN protein & 44 & 84 & 36 & 87 \\
\hline iutA - ferric aerobactin receptor & 66 & 62 & 77 & 46 \\
\hline papC - fimbrial protein & 83 & 45 & 33 & 90 \\
\hline sat - secreted autotransporter toxin & 113 & 15 & 46 & 77 \\
\hline
\end{tabular}

Table 1: A list of various VFs and the number of strains, $N_{i}$, in each of the four states obtained from [4]. Note that $N_{1}+\ldots+N_{4}=251$ for each VF.

From the data given in [4], we choose to analyze 9 VFs. These VFs along with the number of strains, $N_{i}$, in each state $i, i=1, \ldots, 4$, are given in Table 1 . We model this data set by applying a 4-type, continuous time Markov branching process $\boldsymbol{X}(t)=\left(U_{0}, U_{1}, K_{1}, K_{0}\right)^{\prime}$ and branching rates as given in Eq. (1). Figure 1 gives a diagrammatic representation of the parameters used in the model. In the figure, $\lambda_{i}, i=1, \ldots, 4$, represent speciation rates of strains in various states, $q_{12}, q_{21}, q_{34}, q_{43}$ are the transition rates from pathogenic to nonpathogenic states and vice versa, and finally, $m_{32}$ represents migration from state $K_{1}$ to $U_{1}$. As in [4], extinction rates are set to zero for all 4 states, and, pathogenic and nonpathogenic strains are allowed to transition back and forth in the same environment (urinary tract or intestine). It is well known that E. coli travel from the gastrointestinal tract to the bladder in the human host, and cause urinary tract infections ([5], [10]). Hence, we assume that $E$. coli migrate from states $K_{1}$ to $U_{1}$.

All subsequent analysis of the bacterial data set is carried out in $\mathrm{R}$ [15]. The discrete MuSSE model [7] is applied to the data set of each VF to 


\begin{tabular}{|c|c|c|c|c|c|c|c|c|c|}
\hline & $\lambda_{1}$ & $\lambda_{2}$ & $\lambda_{3}$ & $\lambda_{4}$ & $q_{12}$ & $q_{21}$ & $q_{34}$ & $q_{43}$ & $m_{32}$ \\
\hline 1. & $\lambda_{1}$ & $\lambda_{2}$ & $\lambda_{3}$ & $\lambda_{4}$ & $q_{12}$ & $q_{12}$ & $q_{34}$ & $q_{43}$ & $m_{32}$ \\
\hline 2. & $\lambda_{1}$ & $\lambda_{2}$ & $\lambda_{3}$ & $\lambda_{4}$ & $q_{12}$ & $q_{21}$ & $q_{34}$ & $q_{34}$ & $m_{32}$ \\
\hline 3. & $\lambda_{1}$ & $\lambda_{2}$ & $\lambda_{3}$ & $\lambda_{4}$ & $q_{12}$ & $q_{12}$ & $q_{34}$ & $q_{34}$ & $m_{32}$ \\
\hline 4 & $\lambda_{1}$ & $\lambda_{2}$ & $\lambda_{3}$ & $\lambda_{4}$ & 0 & $q_{21}$ & $q_{34}$ & $q_{43}$ & $m_{32}$ \\
\hline 5 & $\lambda_{1}$ & $\lambda_{2}$ & $\lambda_{3}$ & $\lambda_{4}$ & $q_{12}$ & 0 & $q_{34}$ & $q_{43}$ & $m_{32}$ \\
\hline 6 & $\lambda_{1}$ & $\lambda_{2}$ & $\lambda_{3}$ & $\lambda_{4}$ & $q_{12}$ & $q_{21}$ & 0 & $q_{43}$ & $m_{32}$ \\
\hline 7. & $\lambda_{1}$ & $\lambda_{2}$ & $\lambda_{3}$ & $\lambda_{4}$ & $q_{12}$ & $q_{21}$ & $q_{34}$ & 0 & $m_{32}$ \\
\hline 8. & $\lambda_{1}$ & $\lambda_{2}$ & $\lambda_{3}$ & $\lambda_{4}$ & $q_{12}$ & $q_{12}$ & 0 & $q_{43}$ & $m_{32}$ \\
\hline 9. & $\lambda_{1}$ & $\lambda_{2}$ & $\lambda_{3}$ & $\lambda_{4}$ & $q_{12}$ & $q_{12}$ & $q_{34}$ & 0 & $m_{32}$ \\
\hline 10. & $\lambda_{1}$ & $\lambda_{2}$ & $\lambda_{3}$ & $\lambda_{4}$ & 0 & $q_{21}$ & $q_{34}$ & $q_{34}$ & $m_{32}$ \\
\hline 11. & $\lambda_{1}$ & $\lambda_{2}$ & $\lambda_{3}$ & $\lambda_{4}$ & $q_{12}$ & 0 & $q_{34}$ & $q_{34}$ & $m_{32}$ \\
\hline 12. & $\lambda_{1}$ & $\lambda_{1}$ & $\lambda_{3}$ & $\lambda_{4}$ & $q_{12}$ & $q_{21}$ & $q_{34}$ & $q_{43}$ & $m_{32}$ \\
\hline 13. & $\lambda_{1}$ & $\lambda_{2}$ & $\lambda_{3}$ & $\lambda_{3}$ & $q_{12}$ & $q_{21}$ & $q_{34}$ & $q_{43}$ & $m_{32}$ \\
\hline 14. & $\lambda_{1}$ & $\lambda_{1}$ & $\lambda_{3}$ & $\lambda_{4}$ & $q_{12}$ & $q_{12}$ & $q_{34}$ & $q_{43}$ & $m_{32}$ \\
\hline 15. & $\lambda_{1}$ & $\lambda_{2}$ & $\lambda_{3}$ & $\lambda_{3}$ & $q_{12}$ & $q_{21}$ & $q_{34}$ & $q_{34}$ & $m_{32}$ \\
\hline 16. & $\lambda_{1}$ & $\lambda_{1}$ & $\lambda_{3}$ & $\lambda_{3}$ & $q_{12}$ & $q_{12}$ & $q_{34}$ & $q_{34}$ & $m_{32}$ \\
\hline 17. & 0 & $\lambda_{2}$ & $\lambda_{3}$ & $\lambda_{4}$ & $q_{12}$ & $q_{21}$ & $q_{34}$ & $q_{43}$ & $m_{32}$ \\
\hline 18. & $\lambda_{1}$ & 0 & $\lambda_{3}$ & $\lambda_{4}$ & $q_{12}$ & $q_{21}$ & $q_{34}$ & $q_{43}$ & $m_{32}$ \\
\hline 19. & $\lambda_{1}$ & $\lambda_{2}$ & 0 & $\lambda_{4}$ & $q_{12}$ & $q_{21}$ & $q_{34}$ & $q_{43}$ & $m_{32}$ \\
\hline 20. & $\lambda_{1}$ & $\lambda_{2}$ & $\lambda_{3}$ & 0 & $q_{12}$ & $q_{21}$ & $q_{34}$ & $q_{43}$ & $m_{32}$ \\
\hline
\end{tabular}

Table 2: A list of 20 parameter constraints used in the analysis. The first row gives parameters for a model in which no constraints are applied, i.e., all parameters are allowed to vary freely. The subsequent rows represent models in which at least one constraint is used: a parameter is either constrained to be zero, or set equal to another parameter. In each row succeeding the first, which is to be used as a reference row in this table, the constrained parameters are highlighted in bold. For example, in the row marked (1), $q_{21}=q_{12}$, while the remaining parameters are free to vary; in row (2), $q_{43}=q_{34}$, etc.

obtain estimates for all parameters. This is achieved by making use of the 'make.musse()' function in the $\mathrm{R}$ package diversitree [7], which allows for maximum likelihood estimation of the models' parameters. During the parameter estimation analysis of each VF, the initial state for the process is determined by the relative probability of observing type- 3 and type-4 strains, i.e., $\left(0,0, N_{3} /\left(N_{3}+N_{4}\right), N_{4} /\left(N_{3}+N_{4}\right)\right)^{\prime}$. To increase the estimation power of the MuSSE framework, we try out various models in which different constraints are applied on the parameters (the extinction rates already being set to zero), as given in Table 2. From the table, it can be seen that parameters are either set to be zero, or, pairs of parameters are constrained to be equal. This is done by utilizing the 'constrain()' function in the diversitree package. The most suitable constraint on the parameters is then chosen using the Bayesian information criterion or BIC [17], that is, for each VF separately, we choose that combination of constrained (or freely varying) parameter estimates which give the lowest BIC. 


\begin{tabular}{lccccccccc} 
VF & $\lambda_{1}$ & $\lambda_{2}$ & $\lambda_{3}$ & $\lambda_{4}$ & $q_{12}$ & $q_{21}$ & $q_{34}$ & $q_{43}$ & $m_{32}$ \\
\hline astA & 26.39 & 0.000 & 229.6 & 0 & 176.0 & 904.4 & 407.4 & 41.68 & 65.53 \\
\hline cnf1 & 5.840 & 108.3 & 284.8 & 8.066 & 1.185 & 144.6 & 341.3 & 14.92 & 121.5 \\
\hline fimG & 4.105 & 33.75 & 96.99 & 3.737 & 11.74 & 11.74 & 3.715 & 3.715 & 35.18 \\
\hline fyuA & 0.464 & 51.71 & 120.7 & 4.242 & 0 & 38.36 & 47.40 & 6.445 & 46.71 \\
\hline hly1 & 4.630 & 101.7 & 265.6 & 10.58 & 0 & 131.1 & 287.9 & 17.77 & 110.4 \\
\hline iroN & 1.843 & 48.50 & 164.0 & 0 & 21.20 & 60.93 & 178.0 & 20.55 & 51.44 \\
\hline iutA & 0.000 & 64.93 & 126.6 & 3.923 & 49.07 & 157.0 & 43.34 & 5.527 & 46.21 \\
\hline papC & 4.314 & 100.7 & 164.9 & 8.197 & 1.915 & 128.5 & 152.7 & 16.06 & 59.41 \\
\hline sat & 6.059 & 165.6 & 157.4 & 6.493 & 0 & 307.3 & 102.4 & 9.691 & 65.62 \\
\hline
\end{tabular}

Table 3: Parameter values for all VFs in the branching model $\boldsymbol{X}(t)$.

Results The parameter values obtained as a result of the analysis are given in Table 3. We conclude that for 6 out of $9 \mathrm{VFs}$, whenever the parameters are constrained in some manner, the model is a better fit to the given data set, in contrast to when all the parameters are allowed to vary freely. From the parameter values in Table 3, we have that:

(a) $\lambda_{2}>\lambda_{1}$ for 8 out of $9 \mathrm{VFs}$, and $\lambda_{3}>\lambda_{4}$ for all VFs. Thus, virulent strains of $E$. coli, in both urinary and digestive environments, speciate at a higher rate than nonvirulent strains.

(b) $q_{21} \geq q_{12}$ and $q_{34} \geq q_{43}$ for all VFs. Thus, E. coli bacteria, in both digestive and urinary tracts, lose their pathogenicity at a higher rate as compared to gaining it.

Using the mathematical analyses in previous sections and Appendix A, we also find that in accordance with our assumptions (F1) to (F6), $\gamma_{3}>0$ is the largest eigenvalue for all VFs and $\boldsymbol{v}_{\mathbf{3}}>0$ the corresponding right eigenvector. Let $\boldsymbol{v}=\left(\nu_{1}, \ldots, \nu_{4}\right)^{\prime}$ be the normalized version of $\boldsymbol{v}_{\mathbf{3}}$, and let $C=\nu_{1}+\ldots+\nu_{4}$, as before. Then, from Thm. A.4 and using Eq. (2), we have for $N=251$,

$$
\frac{\left(N_{1}, \ldots, N_{4}\right)^{\prime}}{N} \stackrel{a . s .}{\rightarrow} \boldsymbol{p}=\left(p_{1}, \ldots, p_{4}\right)^{\prime},
$$

where $\boldsymbol{p}=\left(p_{1}, \ldots, p_{4}\right)^{\prime}:=\boldsymbol{v} / C$ is the limit of the proportions of E. coli strains. For all VFs, the values of $N_{i} / N$ and $p_{i}, i=1, \ldots, 4$, are given in Table 4 . The sum, $p_{2}+p_{3}$, gives the probability of maintaining each VF in the E. coli strains. From Table 4, we infer that the probability of maintaining VFs varies in the strains; it depends on the VF under consideration. Figure 2 compares the value $p_{2}+p_{3}$, with the sum $N_{2} / N+N_{3} / N$. It can be seen that E. coli strains carrying VFs fimG, fyuA and iut A have a higher probability of being virulent as compared to strains carrying $\mathbf{c n f 1}$, hly1 and sat.

Confidence regions We now apply Thm. A.5 to construct confidence regions for $\boldsymbol{p}$ - the limit of proportions of E. coli strains. Using the expression in Eq. (3), let 


\begin{tabular}{lcccccccc} 
VF & $N_{1} / N$ & $N_{2} / N$ & $N_{3} / N$ & $N_{4} / N$ & $p_{1}$ & $p_{2}$ & $p_{3}$ & $p_{4}$ \\
\hline astA & 0.43 & 0.08 & 0.03 & 0.46 & 0.83 & 0.15 & 0.01 & 0.01 \\
\hline cnf1 & 0.36 & 0.15 & 0.01 & 0.48 & 0.60 & 0.06 & 0.02 & 0.32 \\
\hline fimG & 0.05 & 0.46 & 0.48 & 0.01 & 0.08 & 0.45 & 0.44 & 0.03 \\
\hline fyuA & 0.20 & 0.31 & 0.29 & 0.20 & 0.35 & 0.32 & 0.15 & 0.18 \\
\hline hly1 & 0.35 & 0.16 & 0.02 & 0.47 & 0.51 & 0.08 & 0.04 & 0.37 \\
\hline iroN & 0.18 & 0.33 & 0.14 & 0.35 & 0.52 & 0.35 & 0.02 & 0.11 \\
\hline iutA & 0.26 & 0.25 & 0.31 & 0.18 & 0.34 & 0.20 & 0.23 & 0.23 \\
\hline papC & 0.33 & 0.18 & 0.13 & 0.36 & 0.52 & 0.10 & 0.07 & 0.31 \\
\hline sat & 0.45 & 0.06 & 0.18 & 0.31 & 0.55 & 0.03 & 0.09 & 0.33 \\
\hline
\end{tabular}

Table 4: Limit values, $p_{i}$, for the the branching model $\boldsymbol{X}(t)$, and the proportion of strains, $N_{i} / N$, in each state, $i=1, \ldots, 4$. Here, $N=251, N_{i}$ is the number of strains in each state $i$, and $p_{i}=\nu_{i} / C$, where $\nu_{i}(i=1, \ldots, 4)$ are the components of the normalized eigenvector $\boldsymbol{v}$ and $C=\sum_{i=1}^{4} \nu_{i}$.

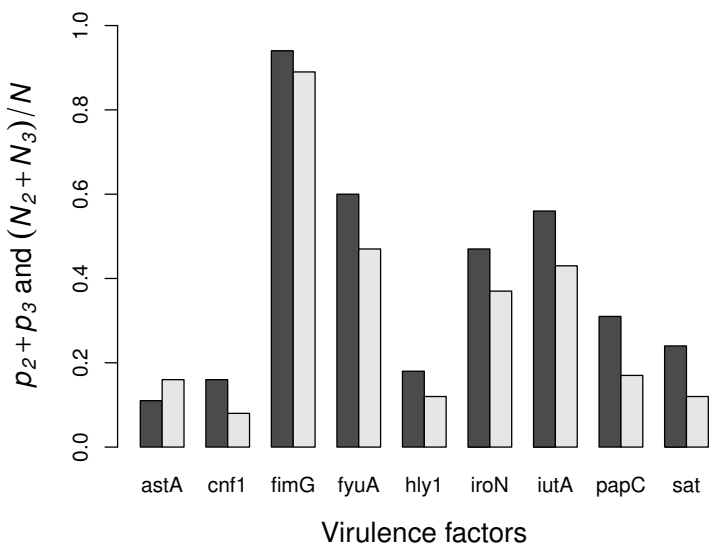

Figure 2: A bar chart comparing the probabilities, $p_{2}+p_{3}$, of maintaining VFs in bacterial strains, with the sum, $\left(N_{2}+N_{3}\right) / N$, of strain proportions. The values used in this figure are obtained from Table $4 . p_{2}+p_{3}$ is represented by grey bars and $\left(N_{2}+N_{3}\right) / N$ by black bars.

$$
D^{2}=N\left(\frac{\widetilde{\boldsymbol{X}}\left(T_{N}\right)}{N}-\frac{\widetilde{\boldsymbol{v}}}{C}\right)^{\prime}\left(\widetilde{\boldsymbol{\Sigma}}_{\boldsymbol{b}}\right)^{-1}\left(\frac{\widetilde{\boldsymbol{X}}\left(T_{N}\right)}{N}-\frac{\widetilde{\boldsymbol{v}}}{C}\right)
$$

where vectors and matrices with the first type removed are denoted by a tilde above them, that is, $\widetilde{\boldsymbol{X}}\left(T_{N}\right)=\left(X_{2}\left(T_{N}\right), \ldots, X_{4}\left(T_{N}\right)\right)^{\prime}=\left(N_{2}, \ldots, N_{4}\right)^{\prime}$, $\widetilde{\boldsymbol{v}}=\left(\nu_{2}, \ldots, \nu_{4}\right)^{\prime}$, and $\widetilde{\boldsymbol{\Sigma}}_{\boldsymbol{b}}=\boldsymbol{\Sigma}_{\boldsymbol{b}}$ with the first row and column removed. Here, it is important to notice that the matrix $\boldsymbol{\Sigma}_{\boldsymbol{b}}$ has rank 3, hence, for the construction of the subsequent confidence regions, we have removed the counts for the first type (alternatively, we can choose to remove any one of the four types) in Eq. (4). From the theory developed in Chapters 4.2 and 5.4 


\begin{tabular}{lccccc} 
VF & $D^{2}$ & $\left(N_{2}, N_{3}, N_{4}\right) / N$ & $a$ & $b$ & $c$ \\
\hline astA & - & - & - & - & - \\
\hline cnf1 & 10.35 & $(0.15,0.01,0.48)$ & 0.651 & 0.091 & 0.046 \\
\hline fimG & 3.421 & $(0.46,0.48,0.01)$ & 0.352 & 0.034 & 0.021 \\
\hline fyuA & 5.655 & $(0.31,0.29,0.20)$ & 0.409 & 0.135 & 0.101 \\
\hline hly1 & 8.963 & $(0.16,0.02,0.47)$ & 0.280 & 0.098 & 0.060 \\
\hline iroN & - & - & - & - & - \\
\hline iutA & 5.989 & $(0.25,0.31,0.18)$ & 0.488 & 0.127 & 0.061 \\
\hline papC & 8.499 & $(0.18,0.13,0.36)$ & 0.362 & 0.097 & 0.081 \\
\hline sat & 6.589 & $(0.06,0.18,0.31)$ & 0.172 & 0.098 & 0.045 \\
\hline
\end{tabular}

Table 5: Specification of confidence ellipsoids for limits of proportions of E. coli strains. For a significance level $\alpha=0.01$, the observed generalized squared distance $D^{2}$, defined by Eq. (4) and (5), is given as $D^{2} \leq \chi_{3}^{2}(0.01)=11.345$. The center of the confidence ellipsoids is represented by $\left(N_{2}, N_{3}, N_{4}\right) / N$, while the half-lengths of the axes are denoted by $a, b$, and $c$.

of [9], at a given significance level $\alpha$, a $100(1-\alpha) \%$ joint confidence region for $\boldsymbol{p}$-which can be thought of as the mean of a multidimensional normal distribution - is defined by ellipsoids such that,

$$
D^{2} \leq \chi_{3}^{2}(\alpha)
$$

where $\chi_{3}^{2}(\alpha)$ is the upper $\alpha$-level quantile of the $\chi^{2}$ distribution with 3 degrees of freedom. The quantity $D^{2}$ represents the square of the generalized distance from the centre of the confidence ellipsoid to a constant density surface. For various VFs, the observed $D^{2}$ value is calculated using Eq. (4), and is given in the second column of Table 5. It can be seen that for astA and iroN, we are unable to find $D^{2}$, since the conditions of Thm. A.5 are not met for these two VFs; the second largest eigenvalue $\gamma_{1}$ is greater than $\gamma_{3} / 2$, i.e., $\gamma_{3} / 2<\gamma_{1}<\gamma_{3}$. In this case, weak convergence is shown but to a random variable, whose distribution is not characterized, only its existence (Corollary 3.18 in [8]).

The axes of the confidence ellipsoids and their relative lengths are determined using the eigenvalues and corresponding eigenvectors of the positive definite matrix $\widetilde{\boldsymbol{\Sigma}}_{\boldsymbol{b}}$ [9]. Since $\widetilde{\boldsymbol{\Sigma}}_{\boldsymbol{b}}$ is a $3 \times 3$ matrix with the first type removed, we can find a simultaneous confidence ellipsoid for $p_{i}$ when $i=2,3$ and 4 . Let $\beta, \zeta$, and $\eta$ be the positive eigenvalues, and $\hat{\beta}, \hat{\zeta}$, and $\hat{\eta}$, the corresponding right eigenvectors of $\widetilde{\boldsymbol{\Sigma}}_{\boldsymbol{b}}$. Then, the axes of the confidence ellipsoids centered at $\widetilde{\boldsymbol{X}}\left(T_{N}\right) / N$, are given as

$$
\pm a \hat{\beta}, \quad \pm b \hat{\zeta}, \quad \text { and } \pm c \hat{\eta}
$$

with $a=D \sqrt{\beta / N}, b=D \sqrt{\zeta / N}$, and $c=D \sqrt{\eta / N}$ being the half length of the three axes. For various VFs, the axes lengths can be computed, as shown in Table 5. 
Since Thm. A.5 is an asymptotic result, and our sample size consists of only 251 data points, we must confirm that Thm. A.5 has been successfully implemented while finding the confidence regions in the aforementioned analysis. To achieve this, we check how the observed proportions $\left(N_{i} / N\right)$ behave for the observed number of strains $(N=251)$ by making use of simulated trees. For the estimated model parameters, given in Table 3, we obtain the clades evolution using the 'tree.musse()' function of the diversitree package in R. For various VFs, excluding astA and iroN, we simulate 10000 trees, each with 251 tips. To ensure that the root of all simulated trees is a dominating type (assumption (F5) in Appendix A), we take the prior distribution to be concentrated on states 3 and 4 , with probabilities equaling the observed relative proportions of types- 3 and 4 . For this, we use the 'sample()' function of $\mathrm{R}$ which randomly selects either of the two states with desired probabilities. Out of the 10000 simulations, we take into consideration in the subsequent analysis, only those trees in which the observed tips have at least one obser-
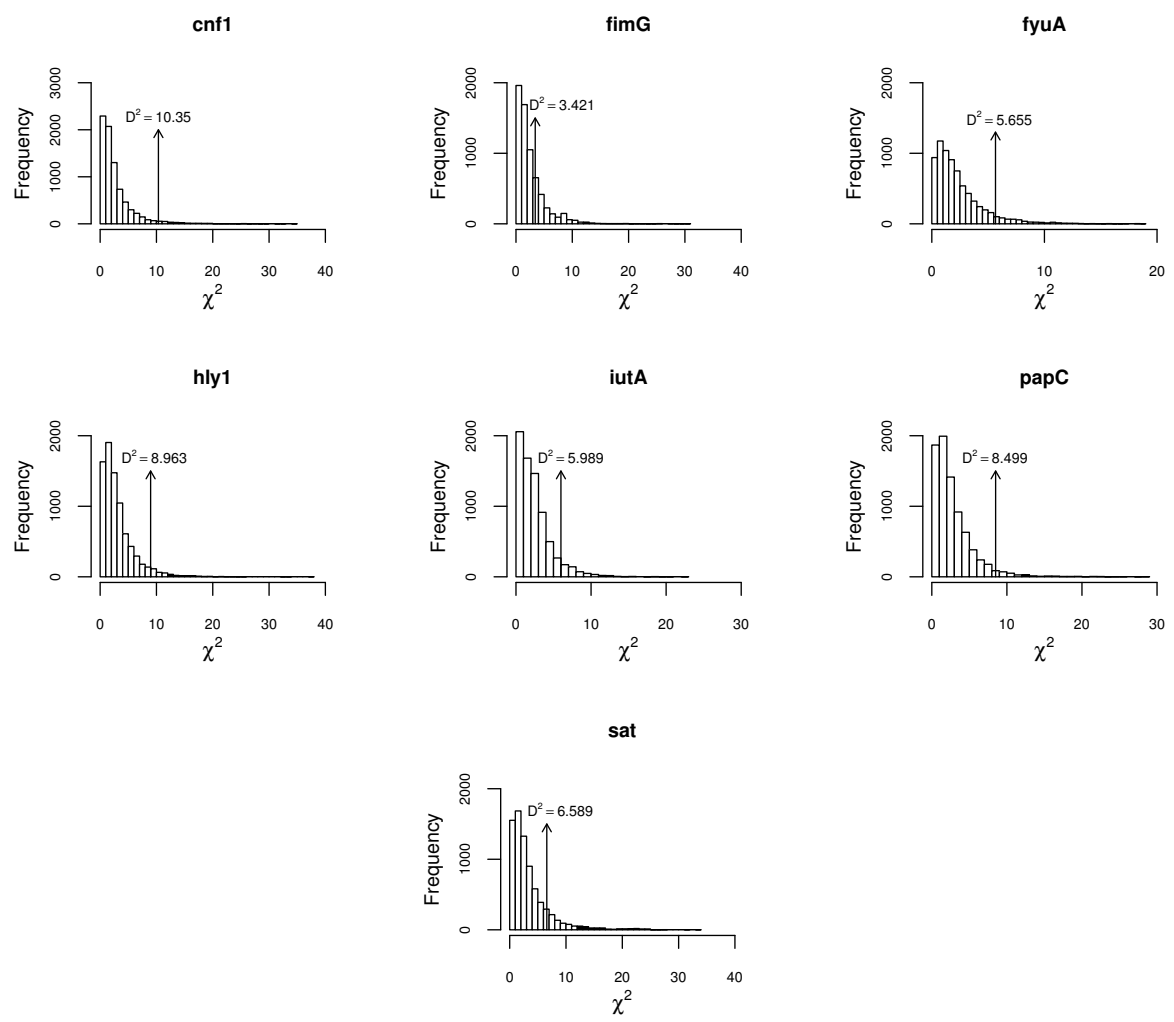

Figure 3: For various VFs, histograms showing the distribution of generalized squared distances for simulated trees. The values less than $\chi_{3}^{2}(0.01)=11.345$ are represented by white bars, and those greater than $\chi_{3}^{2}(0.01)$ are given in black. Arrows in each histogram represent the observed $D^{2}$ value as obtained in Table 5. 


\begin{tabular}{lccc} 
VF & $\mathcal{N}_{\text {sim }}$ & $\mathcal{F}_{\text {sim }}$ & $f_{\text {sim }}$ \\
\hline astA & - & - & - \\
\hline cnf1 & 7958 & 0.031 & 0.02 \\
\hline fimG & 6588 & 0.236 & 0.01 \\
\hline fyuA & 7276 & 0.074 & 0.01 \\
\hline hly1 & 8073 & 0.046 & 0.02 \\
\hline iroN & - & - & - \\
\hline iutA & 7405 & 0.071 & 0.01 \\
\hline papC & 7967 & 0.096 & 0.01 \\
\hline sat & 7583 & 0.128 & 0.03 \\
\hline
\end{tabular}

Table 6: Values obtained from simulating 10000 trees: $\mathcal{N}_{\text {sim }}$ denotes the total number of simulations, out of 10000 , that are actually used in the analysis, $\mathcal{F}_{\text {sim }}$ is that fraction of simulations for which the generalized square distance is greater than the observed $D^{2}$ value (given in Table 5), and $f_{\text {sim }}$ is that fraction of simulations for which the generalized square distance is greater than $\chi_{3}^{2}(0.01)=11.345$.

vation of type-3 or 4 . This is due to the essential nonextinction assumption of Thm. A.5 (cf. Def. A.2). For each VF, the number of simulations that we use out of 10000 is denoted by $\mathcal{N}_{\text {sim }}$, and shown in Table 6. From the 251 tip counts of each simulated tree, we calculate the proportions of types, and then obtain the $D^{2}$ value given in Eq. (4), i.e., the squared generalized distance from the simulated proportions (with the first coordinate removed) which lie on the surface of an ellipsoid, to the ellipsoid's centre (at $\widetilde{\boldsymbol{v}} / C$ ). Using the $\chi^{2}$ distribution with 3 degrees of freedom, we obtain from Eq. (5), how 'far in the tail' the observed $D^{2}$ values lie.

The results are shown in the form of histograms in Figure 3. We conclude that the observed proportions are close to the ellipsoid's centre, i.e., the quantile corresponds to a level greater than a cutoff value of $\alpha=0.01$, and thus for the given number of strains, $N=251$, we are indeed close to the asymptotic regime. From the histograms, we obtain the fraction of simulations, $\mathcal{F}_{\text {sim }}$, which give a value of the generalized square distance greater than the observed value of $D^{2}$, as well as the fraction of simulations, $f_{\text {sim }}$, for which the generalized squared distance is greater than the critical value, $\chi_{3}^{2}(0.01)=11.345$. For each VF, these values are shown in Table 6 .

5. Two variations of the branching model We now model the same E. coli data set [4] by applying another 4-type, Markov branching process $\boldsymbol{Y}(t)=\left(U_{0}, U_{1}, K_{1}, K_{0}\right)^{\prime}$ with branching rates as shown in Figure 4 . All parameters of speciation and transition are the same as in the original process $\boldsymbol{X}(t)$, except for the migration rate, $m_{32}$, which is replaced by $m_{42}$ in this case. Since $E$. coli are assumed to travel from the intestinal to the urinary tract and cause infections, here we assume that bacteria migrate from the nonvirulent state $K_{0}$ to the virulent state $U_{1}$. The mean offspring matrix, $\hat{\boldsymbol{A}}$, 


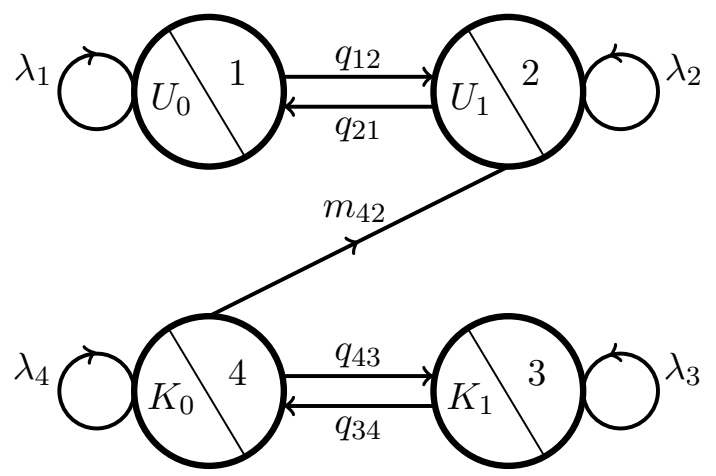

Figure 4: Diagrammatic representation of the branching process $\boldsymbol{Y}(t)$. The four states are similar to the ones in the branching model $\boldsymbol{X}(t)$. The parameters $\lambda_{i}$, $i=1, \ldots, 4$, represent speciation rates in each state, $q_{12}, q_{21}, q_{34}$ and $q_{43}$ are the transition rates between states, $m_{42}$ represents migration from state $K_{0}$ to $U_{1}$.

for this process is given as

$$
\hat{\boldsymbol{A}}=\left(\begin{array}{cccc}
\lambda_{1}-q_{12} & q_{21} & 0 & 0 \\
q_{12} & \lambda_{2}-q_{21} & 0 & m_{42} \\
0 & 0 & \lambda_{3}-q_{34} & q_{43} \\
0 & 0 & q_{34} & \lambda_{4}-q_{43}-m_{42}
\end{array}\right),
$$

with eigenvalues

$$
\begin{gathered}
\hat{\gamma}_{1}=\frac{1}{2}\left(\lambda_{1}-q_{12}+\lambda_{2}-q_{21}+\sqrt{\left(\lambda_{1}-q_{12}-\lambda_{2}+q_{21}\right)^{2}+4 q_{12} q_{21}}\right), \\
\hat{\gamma}_{2}=\frac{1}{2}\left(\lambda_{1}-q_{12}+\lambda_{2}-q_{21}-\sqrt{\left(\lambda_{1}-q_{12}-\lambda_{2}+q_{21}\right)^{2}+4 q_{12} q_{21}}\right), \\
\hat{\gamma}_{3}=\frac{1}{2}\left(\lambda_{3}-m_{42}-q_{34}+\lambda_{4}-q_{43}+\sqrt{\left(\lambda_{3}+m_{42}-q_{34}-\lambda_{4}+q_{43}\right)^{2}+4 q_{34} q_{43}}\right), \\
\hat{\gamma}_{4}=\frac{1}{2}\left(\lambda_{3}-m_{42}-q_{34}+\lambda_{4}-q_{43}-\sqrt{\left(\lambda_{3}+m_{42}-q_{34}-\lambda_{4}+q_{43}\right)^{2}+4 q_{34} q_{43}}\right) .
\end{gathered}
$$

Using Maple 18.00 [13], the left and right column eigenvectors of $\hat{\boldsymbol{A}}$ are obtained as

$$
\begin{aligned}
& \hat{\boldsymbol{u}}_{\mathbf{1}}=\left(\frac{\frac{1}{2}\left(G_{1}+S_{1}\right)\left(\hat{\gamma}_{1} \hat{G}_{3}+\hat{G}_{4}\right)}{q_{21} m_{42} q_{34}}, \frac{\left(\hat{\gamma}_{1} \hat{G}_{3}+\hat{G}_{4}\right)}{q_{34} m_{42}}, 1, \frac{\hat{\gamma}_{1}-\lambda_{3}+q_{34}}{q_{34}}\right)^{\prime}, \\
& \hat{\boldsymbol{u}}_{\mathbf{2}}=\left(\frac{\frac{1}{2}\left(G_{1}-S_{1}\right)\left(\hat{\gamma}_{2} \hat{G}_{3}+\hat{G}_{4}\right)}{q_{21} m_{42} q_{34}}, \frac{\left(\hat{\gamma}_{2} \hat{G}_{3}+\hat{G}_{4}\right)}{q_{34} m_{42}}, 1, \frac{\hat{\gamma}_{2}-\lambda_{3}+q_{34}}{q_{34}}\right)^{\prime}, \\
& \hat{\boldsymbol{u}}_{3}=\left(0,0,1, \frac{1}{2 q_{34}}\left(\hat{G}_{2}+\hat{S}_{2}\right)\right)^{\prime}, \quad \hat{\boldsymbol{u}}_{4}=\left(0,0,1, \frac{1}{2 q_{34}}\left(\hat{G}_{2}-\hat{S}_{2}\right)\right)^{\prime},
\end{aligned}
$$


and

$$
\begin{gathered}
\hat{\boldsymbol{v}}_{\mathbf{1}}=\left(1,-\frac{1}{2 q_{21}}\left(G_{1}-S_{1}\right), 0,0\right)^{\prime}, \quad \hat{\boldsymbol{v}}_{\mathbf{2}}=\left(1,-\frac{1}{2 q_{21}}\left(G_{1}+S_{1}\right), 0,0\right)^{\prime}, \\
\hat{\boldsymbol{v}}_{3}=\left(1, \frac{\hat{\gamma}_{3}-\lambda_{1}+q_{12}}{q_{21}}, \frac{\frac{1}{2}\left(\hat{G}_{2}-\hat{S}_{2}\right)\left(\hat{\gamma}_{3} \hat{G}_{3}+\hat{G}_{4}\right)}{q_{21} m_{42} q_{34}}, \frac{\hat{\gamma}_{3} \hat{G}_{3}+\hat{G}_{4}}{-q_{21} m_{42}}\right)^{\prime}, \\
\hat{\boldsymbol{v}}_{4}=\left(1, \frac{\hat{\gamma}_{4}-\lambda_{1}+q_{12}}{q_{21}}, \frac{\frac{1}{2}\left(\hat{G}_{2}+\hat{S}_{2}\right)\left(\hat{\gamma}_{4} \hat{G}_{3}+\hat{G}_{4}\right)}{q_{21} m_{42} q_{34}}, \frac{\hat{\gamma}_{4} \hat{G}_{3}+\hat{G}_{4}}{-q_{21} m_{42}}\right)^{\prime},
\end{gathered}
$$

respectively, where

$$
\begin{gathered}
\hat{G}_{2}=\delta_{4}-m_{42}-\lambda_{3}+q_{34}, \quad \hat{G}_{3}=\delta_{1}+\delta_{2}-\delta_{4}-\lambda_{3}+q_{34}+m_{42}, \\
\hat{G}_{4}=\lambda_{1} q_{21}-\lambda_{1} \lambda_{2}+\lambda_{2} q_{12}+\lambda_{3} \lambda_{4}-\lambda_{3} m_{42}-\lambda_{3} q_{43}-\lambda_{4} q_{34}+q_{34} m_{42}, \\
\hat{S}_{2}=\sqrt{\left(\hat{G}_{2}\right)^{2}+4 q_{34} q_{43}},
\end{gathered}
$$

while $\delta_{1}, \delta_{2}, \delta_{4}, G_{1}$, and $S_{1}$ are defined previously in Section 3. Similar to the first model $\boldsymbol{X}(t)$, the package diversitree [7] in $\mathrm{R}$ is applied to each VF and estimates of all parameters are obtained, as shown in Table 7. We apply constraints given in Table 2, except that $m_{32}$ is now replaced by $m_{42}$ in the table. The best constraint on the parameters is again chosen using the BIC.

\begin{tabular}{lccccccccc} 
VF & $\lambda_{1}$ & $\lambda_{2}$ & $\lambda_{3}$ & $\lambda_{4}$ & $q_{12}$ & $q_{21}$ & $q_{34}$ & $q_{43}$ & $m_{42}$ \\
\hline astA & 3.031 & 138.9 & 0 & 86.05 & 27.85 & 448.9 & 8.210 & 18.47 & 27.88 \\
\hline cnf1 & 7.054 & 114.8 & 3.608 & 96.15 & 0 & 159.4 & 4.108 & 2.972 & 34.91 \\
\hline fimG & 0.000 & 8.344 & 33.47 & 260.3 & 87.44 & 21.58 & 11.07 & 0 & 553.5 \\
\hline fyuA & 3.192 & 68.53 & 117.9 & 0 & 8.990 & 67.68 & 124.2 & 24.75 & 14.25 \\
\hline hly1 & 4.768 & 109.4 & 0 & 86.64 & 0.823 & 145.7 & 8.042 & 17.18 & 27.30 \\
\hline iroN & 0.875 & 48.39 & 4.562 & 107.5 & 0 & 31.93 & 6.113 & 31.19 & 42.69 \\
\hline iutA & 0 & 75.26 & 157.2 & 7.472 & 36.64 & 158.8 & 104.9 & 3.066 & 12.50 \\
\hline papC & 4.046 & 113.5 & 147.7 & 0 & 2.051 & 140.7 & 201.5 & 26.92 & 13.11 \\
\hline sat & 5.793 & 173.1 & 0 & 107.1 & 0.000 & 318.2 & 14.71 & 65.11 & 34.35 \\
\hline
\end{tabular}

Table 7: Parameter values for the branching process $\boldsymbol{Y}(t)$.

\begin{tabular}{lcccc} 
VF & $\hat{p}_{1}$ & $\hat{p}_{2}$ & $\hat{p}_{3}$ & $\hat{p}_{4}$ \\
\hline astA & 0.42 & 0.06 & 0.14 & 0.38 \\
\hline cnf1 & 0.43 & 0.14 & 0.02 & 0.41 \\
\hline fimG & 0.09 & 0.46 & 0.44 & 0.01 \\
\hline fyuA & 0.34 & 0.21 & 0.17 & 0.28 \\
\hline hly1 & 0.43 & 0.12 & 0.11 & 0.34 \\
\hline iroN & 0.31 & 0.36 & 0.15 & 0.18 \\
\hline iutA & 0.13 & 0.08 & 0.30 & 0.49 \\
\hline papC & 0.51 & 0.09 & 0.10 & 0.30 \\
\hline sat & 0.50 & 0.04 & 0.28 & 0.18 \\
\hline
\end{tabular}

Table 8: Limit values $\hat{p}_{i}(i=1, \ldots, 4)$ for the the branching model $\boldsymbol{Y}(t)$. 


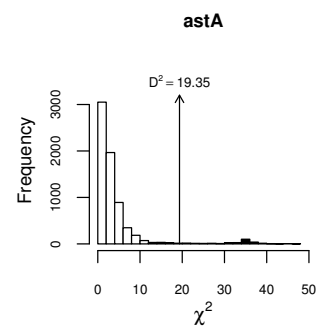

hly1

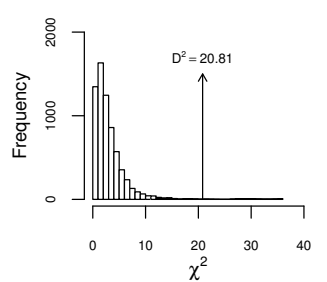

cnf1

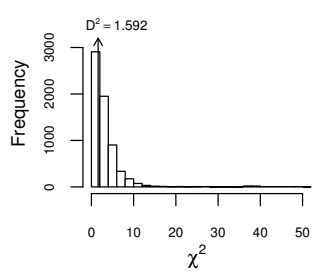

iroN

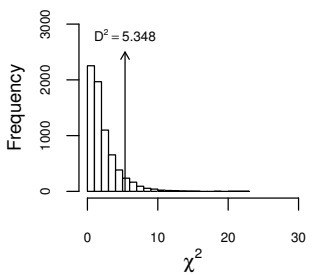

fimG

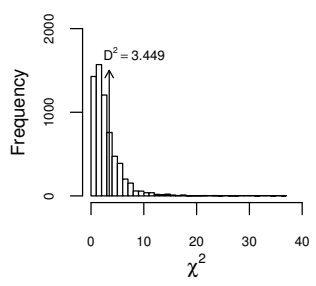

iutA

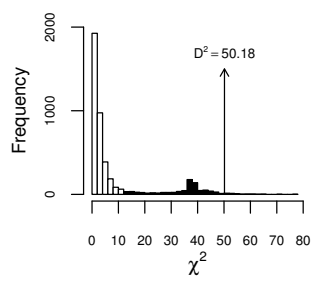

papC

sat
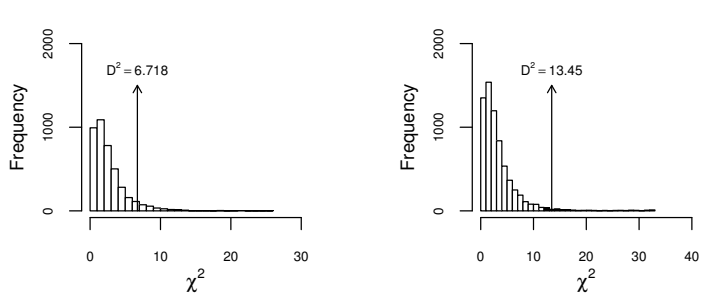

Figure 5: Distribution of generalized squared distances for those VFs for which assumptions of Thm. A.5 hold under the model $\boldsymbol{Y}(t)$. The values less than $\chi_{3}^{2}(0.01)=$ 11.345 are represented by white bars, and those greater than 11.345 are given in black. Arrows in each histogram represent the observed $D^{2}$ value.

\begin{tabular}{lccc} 
VF & $\hat{\mathcal{N}}_{\text {sim }}$ & $\hat{\mathcal{F}}_{\text {sim }}$ & $\hat{f}_{\text {sim }}$ \\
\hline astA & 6885 & 0.040 & 0.05 \\
\hline cnf1 & 6473 & 0.647 & 0.02 \\
\hline fimG & 6476 & 0.291 & 0.02 \\
\hline fyuA & - & - & - \\
\hline hly1 & 6777 & 0.010 & 0.03 \\
\hline iroN & 7012 & 0.080 & 0.00 \\
\hline iutA & 4473 & 0.010 & 0.19 \\
\hline papC & 4157 & 0.064 & 0.01 \\
\hline sat & 6776 & 0.02 & 0.03 \\
\hline
\end{tabular}

Table 9: Results of the confidence regions' analysis for $\hat{\boldsymbol{p}}$ corresponding to the process $\boldsymbol{Y}(t) . \hat{\mathcal{N}}_{\text {sim }}$ represents that number of simulated trees (out of 10000) in which the observed tips have at least a single observation of the dominating type- 3 or 4 . $\hat{\mathcal{F}}_{\text {sim }}$ is the fraction of simulations for which the generalized squared distance is greater than the observed $D^{2}$ value. $\hat{f}_{\text {sim }}$ is that fraction of simulations for which the generalized squared distance is greater than $\chi_{3}^{2}(0.01)=11.345$. 
For each VF, $\hat{\gamma}_{3}$ is found to be the largest eigenvalue, with corresponding normalized eigenvector, say $\hat{\boldsymbol{v}}$. We again apply Thm. A.4 and using the estimated parameters, obtain the limiting values $\hat{\boldsymbol{p}}=\left(\hat{p}_{1}, \ldots, \hat{p}_{4}\right)^{\prime}=\hat{\boldsymbol{v}} / C$, where $C$ is the sum of coordinates of $\hat{\boldsymbol{v}}$. The values of $\hat{\boldsymbol{p}}$ are given in Table 8 . From Tables 7 and 8, we infer that: (a) $\lambda_{2}>\lambda_{1}$ for all VFs and $\lambda_{3}<\lambda_{4}$ for 6 out of $9 \mathrm{VFs}$, (b) $q_{21}>q_{12}$ for 8 out of $9 \mathrm{VFs}$ and $q_{34}>q_{43}$ for 5 out of $9 \mathrm{VFs}$, (c) the probability, $\hat{p}_{2}+\hat{p}_{3}$, of prevalence of VFs in E. coli strains varies with each VF-for instance, the VF fimG has the maximum probability of being maintained. An analysis of the confidence regions for $\hat{\boldsymbol{p}}$ is also carried out, similar to the one for $\boldsymbol{p}$ in Section 4. Figure 5 and Table 9 give the results for this analysis.

We apply yet another 4 -type branching process $\boldsymbol{Z}(t)=\left(U_{0}, U_{1}, K_{1}, K_{0}\right)^{\prime}$ to the same data set. The branching rates are as shown in Figure 6. This time we include migration from both virulent and nonvirulent states in the gastrointestinal tract to the virulent state of the urinary tract, using parameters $m_{32}$ and $m_{42}$. The mean offspring matrix $\overline{\boldsymbol{A}}$ is given as

$$
\overline{\boldsymbol{A}}=\left(\begin{array}{cccc}
\lambda_{1}-q_{12} & q_{21} & 0 & 0 \\
q_{12} & \lambda_{2}-q_{21} & m_{32} & m_{42} \\
0 & 0 & \lambda_{3}-q_{34}-m_{32} & q_{43} \\
0 & 0 & q_{34} & \lambda_{4}-q_{43}-m_{42}
\end{array}\right)
$$

The eigenvalues of $\overline{\boldsymbol{A}}$ are

$$
\begin{gathered}
\bar{\gamma}_{1}=\frac{1}{2}\left(\lambda_{1}-q_{12}+\lambda_{2}-q_{21}+\sqrt{\left(\lambda_{1}-q_{12}-\lambda_{2}+q_{21}\right)^{2}+4 q_{12} q_{21}}\right), \\
\bar{\gamma}_{2}=\frac{1}{2}\left(\lambda_{1}-q_{12}+\lambda_{2}-q_{21}-\sqrt{\left(\lambda_{1}-q_{12}-\lambda_{2}+q_{21}\right)^{2}+4 q_{12} q_{21}}\right), \\
\bar{\gamma}_{3}=\frac{1}{2}\left(\lambda_{3}-m_{32}-q_{34}+\lambda_{4}-m_{42}-q_{43}+\sqrt{\left(\lambda_{3}-m_{32}-q_{34}-\lambda_{4}+m_{42}+q_{43}\right)^{2}+4 q_{34} q_{43}}\right),
\end{gathered}
$$

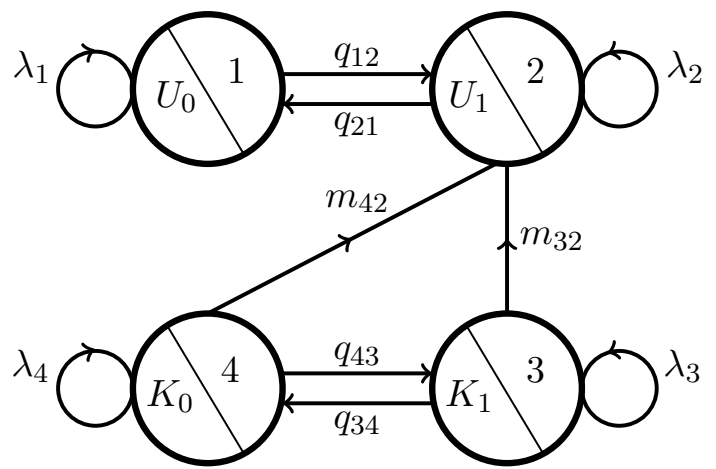

Figure 6: Branching rates for the model $\boldsymbol{Z}(t) . \lambda_{i}, i=1, \ldots, 4$, represent speciation rates, $q_{12}, q_{21}, q_{34}$ and $q_{43}$ are the rates of transition between states, $m_{32}$ and $m_{42}$ are migration rates from state $K_{1}$ to $U_{1}$ and $K_{0}$ to $U_{1}$, respectively. 
$\bar{\gamma}_{4}=\frac{1}{2}\left(\lambda_{3}-m_{32}-q_{34}+\lambda_{4}-m_{42}-q_{43}-\sqrt{\left(\lambda_{3}-m_{32}-q_{34}-\lambda_{4}+m_{42}+q_{43}\right)^{2}+4 q_{34} q_{43}}\right)$.

The left and right column eigenvectors of $\overline{\boldsymbol{A}}$ are obtained as

$$
\begin{gathered}
\overline{\boldsymbol{u}}_{1}=\left(\frac{\frac{1}{2}\left(G_{1}+S_{1}\right)\left(\bar{\gamma}_{1} \bar{G}_{3}+\bar{G}_{4}\right)}{q_{21} G_{5}}, \frac{\bar{\gamma}_{1} \bar{G}_{3}+\bar{G}_{4}}{G_{5}}, 1, \frac{G_{6}}{G_{5}}\right)^{\prime}, \\
\overline{\boldsymbol{u}}_{2}=\left(\frac{-\frac{1}{2}\left(-G_{1}+S_{1}\right)\left(\bar{\gamma}_{2} \bar{G}_{3}+\bar{G}_{4}\right)}{q_{21} G_{5_{2}}}, \frac{\bar{\gamma}_{2} \bar{G}_{3}+\bar{G}_{4}}{G_{5_{2}}}, 1, \frac{G_{6_{2}}}{G_{5_{2}}}\right)^{\prime}, \\
\overline{\boldsymbol{u}}_{\mathbf{3}}=\left(0,0,1, \frac{1}{2 q_{34}}\left(\bar{G}_{2}+\bar{S}_{2}\right)\right)^{\prime}, \quad \overline{\boldsymbol{u}}_{4}=\left(0,0,1, \frac{1}{2 q_{34}}\left(\bar{G}_{2}-\bar{S}_{2}\right)\right)^{\prime} .
\end{gathered}
$$

and

$$
\begin{gathered}
\overline{\boldsymbol{v}}_{\mathbf{1}}=\left(1,-\frac{1}{2 q_{21}}\left(G_{1}-S_{1}\right), 0,0\right)^{\prime}, \quad \overline{\boldsymbol{v}}_{\mathbf{2}}=\left(1,-\frac{1}{2 q_{21}}\left(G_{1}+S_{1}\right), 0,0\right)^{\prime}, \\
\overline{\boldsymbol{v}}_{\mathbf{3}}=\left(1, \frac{\bar{\gamma}_{3}-\lambda_{1}+q_{12}}{q_{21}}, \frac{\left(\bar{\gamma}_{3} \bar{G}_{3}+\bar{G}_{4}\right) G_{8}}{q_{21} G_{7}}, \frac{-\left(\bar{\gamma}_{3} \bar{G}_{3}+\bar{G}_{4}\right) G_{9}}{q_{21} G_{7}}\right)^{\prime}, \\
\overline{\boldsymbol{v}}_{4}=\left(1, \frac{\bar{\gamma}_{4}-\lambda_{1}+q_{12}}{q_{21}}, \frac{\left(\bar{\gamma}_{4} \bar{G}_{3}+\bar{G}_{4}\right) G_{82}}{q_{21} G_{7}}, \frac{-\left(\bar{\gamma}_{4} \bar{G}_{3}+\bar{G}_{4}\right) G_{9_{2}}}{q_{21} G_{7}}\right)^{\prime} .
\end{gathered}
$$

respectively, where

$$
\begin{aligned}
& \bar{G}_{2}=d_{4}-\delta_{3}, \quad \bar{G}_{3}=\delta_{1}+\delta_{2}-\delta_{3}-d_{4}, \\
& \bar{G}_{4}=\hat{G}_{4}-m_{32} d_{4}, \quad \bar{S}_{2}=\sqrt{\left(\bar{G}_{2}\right)^{2}+4 q_{34} q_{43}}, \\
& G_{5}=m_{32}\left(\bar{\gamma}_{1}-d_{4}\right)+m_{42} q_{34}, \quad G_{5_{2}}=m_{32}\left(\bar{\gamma}_{2}-d_{4}\right)+m_{42} q_{34}, \\
& G_{6}=m_{42} \bar{\gamma}_{1}-m_{42} \lambda_{3}+m_{32} m_{42}+m_{32} q_{43}+m_{42} q_{34}, \\
& G_{6_{2}}=m_{42} \bar{\gamma}_{2}-m_{42} \lambda_{3}+m_{32} m_{42}+m_{32} q_{43}+m_{42} q_{34}, \\
& G_{7}=m_{42}^{2} q_{34}-m_{32}^{2} q_{43}-m_{32} m_{42} g_{2}, \\
& G_{8}=m_{42}\left(-\bar{\gamma}_{3}+d_{4}\right)+m_{32} q_{43}, \quad G_{8_{2}}=m_{42}\left(-\bar{\gamma}_{4}+d_{4}\right)+m_{32} q_{43} \\
& G_{9}=m_{32}\left(-\bar{\gamma}_{3}+\delta_{3}\right)+m_{42} q_{34}, \quad G_{9_{2}}=m_{32}\left(-\bar{\gamma}_{4}+\delta_{3}\right)+m_{42} q_{34},
\end{aligned}
$$

with $d_{4}=\lambda_{4}-q_{43}-m_{42}$ and $\delta_{1}, \delta_{2}, \delta_{3}, G_{1}, S_{1}, \hat{G}_{4}$ as defined in earlier sections. Model analysis is carried out in $\mathrm{R}$, and as before, the initial state is determined by the relative probability of observing type- 3 and type- 4 strains. We use the same constraints given in Table 2, except that both $m_{32}$ and $m_{42}$ are allowed to vary. In addition, we use 21 more constraints in which the constraints from Table 2 are repeated using $m_{32}=m_{42}$. The best constraints are chosen according to BIC, and the parameter values obtained are given in Table 10. From the parameters, we compute the eigenvalues and corresponding eigenvectors of $\overline{\boldsymbol{A}}$. $\bar{\gamma}_{3}$ is found to be the largest eigenvalue for all VFs. Moreover, $\overline{\boldsymbol{p}}=\left(\bar{p}_{1}, \ldots, \bar{p}_{4}\right)^{\prime}$, denoting the limits of proportions of E. coli 


\begin{tabular}{lcccccccccc} 
VF & $\lambda_{1}$ & $\lambda_{2}$ & $\lambda_{3}$ & $\lambda_{4}$ & $q_{12}$ & $q_{21}$ & $q_{34}$ & $q_{43}$ & $m_{32}$ & $m_{42}$ \\
\hline astA & 3.031 & 138.8 & 0 & 86.05 & 27.86 & 448.8 & 8.210 & 18.47 & 0.000 & 27.88 \\
\hline cnf1 & 0 & 24.84 & 3.768 & 107.5 & 2.343 & 136.6 & 2.027 & 2.706 & 2.053 & 33.95 \\
\hline fimG & 4.487 & 35.10 & 103.2 & 4.034 & 0 & 11.17 & 1.832 & 2.014 & 30.41 & 2.125 \\
\hline fyuA & 3.705 & 57.57 & 116.2 & 0 & 8.254 & 56.65 & 69.75 & 12.72 & 36.23 & 0.000 \\
\hline hly1 & 4.768 & 109.4 & 0 & 86.64 & 0.823 & 145.7 & 8.046 & 17.18 & 0.000 & 27.30 \\
\hline iroN & 1.902 & 48.60 & 164.7 & 0 & 20.77 & 60.34 & 181.2 & 20.79 & 51.59 & 0.000 \\
\hline iutA & 0 & 71.13 & 155.1 & 7.056 & 38.11 & 148.3 & 47.56 & 1.058 & 9.561 & 9.561 \\
\hline papC & 4.493 & 99.45 & 166.2 & 0 & 2.081 & 126.5 & 203.0 & 25.97 & 49.39 & 0.027 \\
\hline sat & 6.003 & 164.8 & 141.0 & 0 & 0.000 & 299.9 & 124.1 & 17.00 & 44.77 & 0.000 \\
\hline
\end{tabular}

Table 10: Parameter estimates for the branching model $\boldsymbol{Z}(t)$.

\begin{tabular}{lcccc} 
VF & $\bar{p}_{1}$ & $\bar{p}_{2}$ & $\bar{p}_{3}$ & $\bar{p}_{4}$ \\
\hline astA & 0.42 & 0.06 & 0.14 & 0.38 \\
\hline cnf1 & 0.22 & 0.12 & 0.02 & 0.64 \\
\hline fimG & 0.06 & 0.36 & 0.56 & 0.02 \\
\hline fyuA & 0.42 & 0.26 & 0.12 & 0.20 \\
\hline hly1 & 0.42 & 0.12 & 0.11 & 0.35 \\
\hline iroN & 0.55 & 0.37 & 0.01 & 0.06 \\
\hline iutA & 0.07 & 0.06 & 0.59 & 0.28 \\
\hline papC & 0.56 & 0.09 & 0.07 & 0.28 \\
\hline sat & 0.52 & 0.03 & 0.11 & 0.34 \\
\hline
\end{tabular}

Table 11: Limits of proportions of $E$. coli strains for the branching process $\boldsymbol{Z}(t)$.

\begin{tabular}{lccc} 
VF & $\overline{\mathcal{N}}_{\text {sim }}$ & $\overline{\mathcal{F}}_{\text {sim }}$ & $\bar{f}_{\text {sim }}$ \\
\hline astA & 6757 & 0.042 & 0.06 \\
\hline cnf1 & 6810 & 0.004 & 0.01 \\
\hline fimG & 6996 & 0.182 & 0.03 \\
\hline fyuA & - & - & - \\
\hline hly1 & 6845 & 0.012 & 0.02 \\
\hline iroN & - & - & - \\
\hline iutA & 5421 & 0.129 & 0.15 \\
\hline papC & 7033 & 0.023 & 0.01 \\
\hline sat & 6733 & 0.110 & 0.04 \\
\hline
\end{tabular}

Table 12: For the model $\boldsymbol{Z}(t), \overline{\mathcal{N}}_{\text {sim }}$ represents the number of simulations (out of 10000 ) in which at least one observation of the dominating type- 3 or 4 was obtained, $\overline{\mathcal{F}}_{\text {sim }}$ gives that fraction of simulations for which the generalized squared distance is greater than the observed value of $D^{2}$, and $\bar{f}_{\text {sim }}$ is that fraction of simulations for which the generalized squared distance is greater than $\chi_{3}^{2}(0.01)=11.345$.

strains and calculated using Thm. A.4, is given in Table 11. From the tables, we conclude that: (a) $\lambda_{2}>\lambda_{1}$ for all VFs and $\lambda_{3}>\lambda_{4}$ for 6 out of 9 VFs. (b) $q_{21}>q_{12}$ and $q_{34} \geq q_{43}$ for 6 VFs. (c) $m_{32} \geq m_{42}$ for 6 out of 9 VFs. The sum $\bar{p}_{2}+\bar{p}_{3}$ gives the probability of prevalence of various VFs in E. coli strains. An analysis regarding the confidence regions for the limits of strain proportions was also performed, similar to the one for the previous models. 

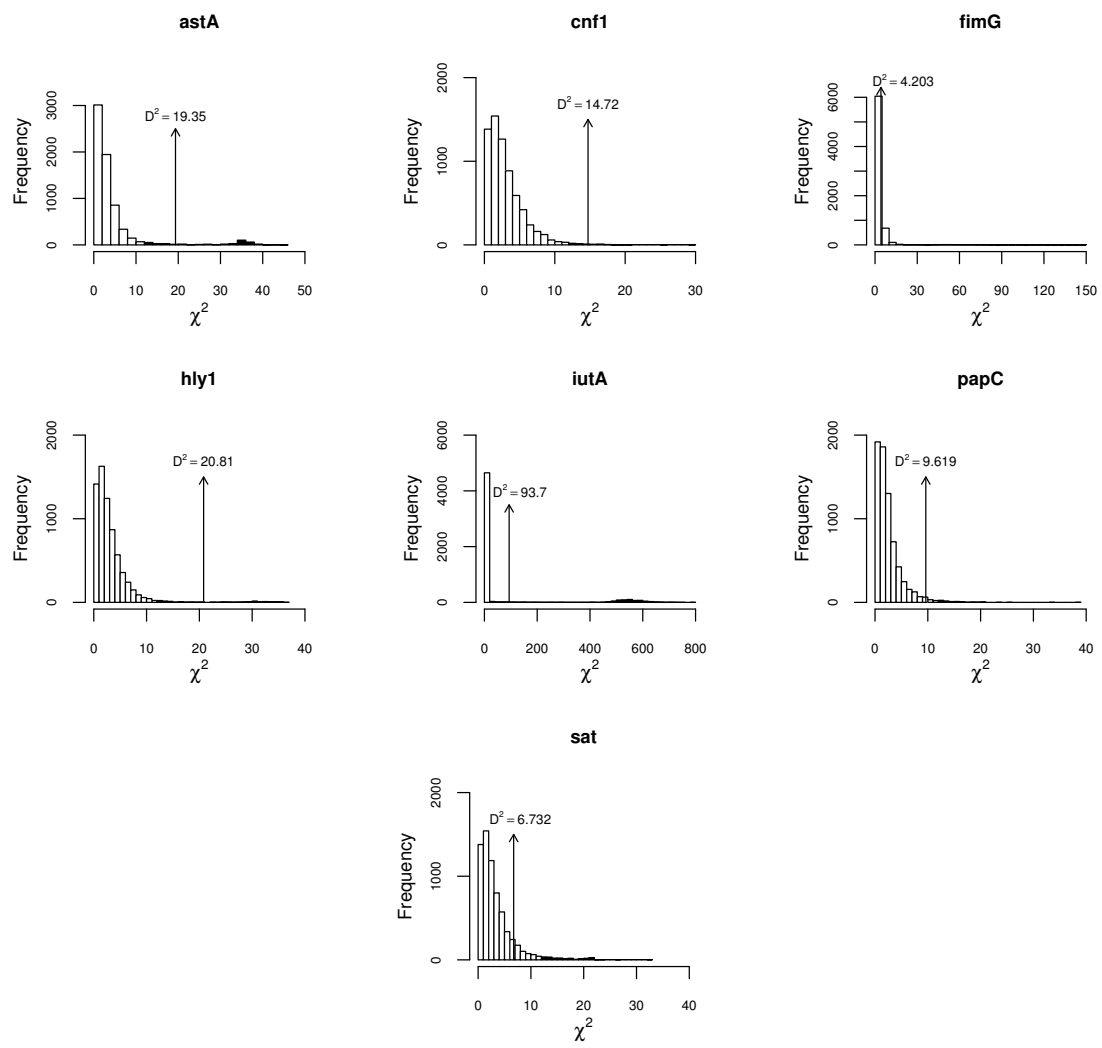

Figure 7: For the process $\boldsymbol{Z}(t)$, these histograms give the distribution of the generalized squared distances (obtained from Eq.(4)) of 10000 simulated trees for those VFs for which Thm. A.5 is satisfied. The values less than $\chi_{3}^{2}(\alpha)=11.345$, for $\alpha=0.01$, are represented by white bars, while the remaining are given in black. Arrowed lines on each histogram represent the observed $D^{2}$ value for each VF.

The results are displayed in Figure 7 and Table 12.

6. Conclusions We now list some concise but useful inferences drawn from the mathematical analysis of the three models. Here, we would also like to state that in a future work, we plan to carry out a rigorous analysis of a larger data set of E. coli strains, which would not only add to the current results, but also lead to more comprehensive and solid biological conclusions.

1. E. coli strains carrying a VF speciate at faster rates as compared to strains which do not carry a VF, in urinary tracts of the human host (Tables 3, 7 and 10). This result remains fairly constant in all three models. A similar result was also obtained in [4], where it was shown that speciation rates were higher for virulent $E$. coli strains as compared to nonvirulent strains that were isolated from human urine samples. The same result, however, does not hold for strains in the digestive tract; according to the process $\boldsymbol{X}(t)$ and 
$\boldsymbol{Z}(t)$, most virulent bacterial strains speciate faster than nonvirulent strains, but the opposite is true under the model $\boldsymbol{Y}(t)$.

2. E. coli bacteria lose their virulence at a higher rate as compared to gaining it, in both urinary and digestive tracts of the human hosts. Under all three models, this behavior is exhibited for majority of the VFs (Tables 3, 7 and 10). This is consistent with the fact that bacteria maintain their virulence only if conditions are favorable for host invasion or colonization, otherwise, they lose their pathogenicity, since the expression of VFs is costly to maintain and tends to decrease bacterial fitness [4, 11].

3. Pathogenic and nonpathogenic bacteria in the gut migrate to the urinary tract at different rates. This result is inferred from the analysis of the third branching model $\boldsymbol{Z}(t)$ with two migration rates (Table 10 ). For 5 out of 9 VFs, pathogenic bacteria are found to migrate faster than the nonpathogenic ones. However, compared separately, from Tables 3 and $7, m_{32}>m_{42}$ for most VFs ( 8 out of 9 ).

4. The probability of maintaining virulence in bacterial strains $\left(p_{2}+p_{3}, \hat{p}_{2}+\hat{p}_{3}\right.$ and $\bar{p}_{2}+\bar{p}_{3}$ in the models $\boldsymbol{X}(t), \boldsymbol{Y}(t)$ and $\boldsymbol{Z}(t)$, respectively) varies with the VF under consideration-see Tables 4, 8 and 11. However, under all three branching models, it is consistently seen that the VF fimG has the highest chance of prevailing in virulent bacterial strains.

5. The confidence region analysis of the limits of proportions of E. coli strains shows that Thm. A.5 is more successfully implemented to the data set under the first two models, $\boldsymbol{X}(t)$ and $\boldsymbol{Y}(t)$, as compared to the third model, $\boldsymbol{Z}(t)$. From figures 3, 5, and 7 we conclude that for Thm.A.5, the branching process $\boldsymbol{X}(t)$ is a better fit for analyzing strains carrying ast A, fimG, fyuA, hly $\mathbf{1}$, iut $\mathbf{A}$, and sat, while $\boldsymbol{Y}(t)$ is suitable for $\mathbf{c n f 1}$, iroN and papC.

A. Appendix In this section, we state the limit theorems that are applied in this paper for the analysis of bacterial strains data. We first list some important definitions and assumptions that relate to $n$-type branching processes.

Definition A.1 A type- $p$ is said to be dominating if it is possible to obtain every other type, say type- $q(q=1, \ldots, n$, and $q \neq p)$, in a branching process that starts with a single type- $p$ particle. The set of all dominating types is called the dominating class [8].

DEFINITION A.2 A branching process becomes essentially extinct if there are no particles from the dominating class at some time instance [8].

\section{Assumptions:}

(F1) Following a branching event involving a type- $p$ particle, the number of type- $p$ particles either increases, or decreases by at most one, while the number of type $-q$ particles, $q=1, \ldots, n, q \neq p$, always increases. 
(F2) Following a branching event involving a type- $p$ particle, all changes in the number of type- $q$ particles, $q=1, \ldots, n$, have finite means and variances, that is, the process never explodes.

(F3) The largest eigenvalue, $\gamma$, of the mean offspring matrix $\boldsymbol{A}$ is positive, i.e., the multitype branching process is supercritical, hence, there exists a positive probability of nonextinction.

(F4) The largest eigenvalue of $\boldsymbol{A}$ is simple, i.e., the algebraic multiplicity of $\gamma$ is one.

(F5) There exists a dominating type- $p$, such that the multitype branching process starts with at least one particle of type- $p$.

(F6) The largest eigenvalue, $\gamma$, belongs to the dominating class.

Theorem A.3 (Theorem 1 in [2]) For the branching process $\boldsymbol{X}(t)$,

$$
\lim _{t \rightarrow \infty} e^{-\gamma t} \boldsymbol{X}(t)=W \boldsymbol{v}
$$

exists with probability 1 , where $W$ is a nonnegative random variable, $\gamma$ is the largest eigenvalue of the mean offspring matrix $\boldsymbol{A}$, and $\boldsymbol{v}$ is the normalized right eigenvector of $\boldsymbol{A}$ corresponding to $\gamma$.

Let $\boldsymbol{b} \in \mathbb{R}^{n}$ be a fixed column vector and let $N \geq 0$. Define

$$
T_{\boldsymbol{b}}(N)=\min \{t \geq 0: \boldsymbol{b} \cdot \boldsymbol{X}(t) \geq N\}
$$

to be the first time when $\boldsymbol{b} \cdot \boldsymbol{X}(t)$ exceeds $N$. Also let $\boldsymbol{b} \cdot \boldsymbol{v}>0$. Then, conditioned on essential nonextinction, $T_{\boldsymbol{b}}(N)<\infty$ for all $N \geq 0$ and $\boldsymbol{b}$. $\boldsymbol{X}(t) \stackrel{\text { a.s. }}{\longrightarrow} \infty$ as $t \rightarrow \infty$ (Lemma 3.14 in [8]).

We now state two more limit theorems, which are applied in the main sections of the paper with the special case $\boldsymbol{b}=(1,1, \ldots, 1) \in \mathbb{R}^{n}$.

Theorem A.4 (Theorem 3.15 in [8]) Assume (F1) to (F6) and let $\boldsymbol{b} \cdot \boldsymbol{v}>0$. Then, conditioned on essential nonextinction, as $N \rightarrow \infty$,

$$
\frac{\boldsymbol{X}\left(T_{\boldsymbol{b}}(N)\right)}{N} \stackrel{a . s .}{\longrightarrow} \frac{\boldsymbol{v}}{\boldsymbol{b} \cdot \boldsymbol{v}} .
$$

Let $\Delta$ be the set of all eigenvalues, $\gamma_{i}$, of $\boldsymbol{A}$. Of course, the largest eigenvalue $\gamma$ also belongs to $\Delta$. There exist projection matrices $\boldsymbol{P}_{\gamma_{i}}$, such that $\sum_{\gamma_{i} \in \Delta} \boldsymbol{P}_{\gamma_{i}}=\boldsymbol{I}$, where $\boldsymbol{I}$ is the identity matrix. Let $\boldsymbol{P}$ be the projection matrix onto the sum of the eigenspaces corresponding to $\gamma_{i}<\gamma / 2$, that is, $\boldsymbol{P}=\sum_{\gamma_{i} \in \Delta_{1}} \boldsymbol{P}_{\gamma_{i}}$, where $\Delta_{1}=\left\{\gamma_{i} \in \Delta: \gamma_{i}<\gamma / 2\right\}$. Also, let $\xi_{i}=\left(\xi_{i 1}, \ldots, \xi_{i n}\right)^{\prime}$ be a random column vector with integer coordinates, denoting the change in population if a branching event occurs at a particle 
of type- $i$, and define the matrix $\boldsymbol{B}$ as $\boldsymbol{B}=\sum_{i=1}^{n} \nu_{i} a_{i} \boldsymbol{B}_{i}$, where $\nu_{i}$ represents the coordinates of the eigenvector $\boldsymbol{v}$ and $\boldsymbol{B}_{i}=\mathbb{E}\left(\xi_{i} \xi_{i}^{\prime}\right)$. Furthermore, define another matrix $\boldsymbol{\Sigma}_{\boldsymbol{I}}$ as

$$
\boldsymbol{\Sigma}_{\boldsymbol{I}}=\int_{0}^{\infty} \boldsymbol{P} e^{s \boldsymbol{A}} \boldsymbol{B}\left(\boldsymbol{P} e^{s \boldsymbol{A}}\right)^{\prime} e^{-s \gamma} d s
$$

where $s \in \mathbb{R}[8]$.

Theorem A.5 (Corollary 3.16 in/81) Assume (F1) to (F6) and $\boldsymbol{b} \cdot \boldsymbol{v}>0$. Suppose further that $\gamma / 2>\gamma_{(n-1)}$, where $\gamma_{(n-1)}$ is the second largest eigenvalue, i.e., the second largest eigenvalue is less than half the largest eigenvalue. Then, conditioned on essential nonextinction, as $N \rightarrow \infty$,

$$
\sqrt{N}\left(\frac{\boldsymbol{X}\left(T_{\boldsymbol{b}}(N)\right)}{N}-\frac{\boldsymbol{v}}{\boldsymbol{b} \cdot \boldsymbol{v}}\right) \stackrel{\mathcal{D}}{\longrightarrow} \mathcal{N}\left(0, \boldsymbol{\Sigma}_{\boldsymbol{b}}\right)
$$

where

$$
\boldsymbol{\Sigma}_{\boldsymbol{b}}=(\boldsymbol{b} \cdot \boldsymbol{v})^{-1}\left(\boldsymbol{I}-\frac{\boldsymbol{v} \boldsymbol{b}^{\prime}}{\boldsymbol{b} \cdot \boldsymbol{v}}\right) \boldsymbol{\Sigma}_{\boldsymbol{I}}\left(\boldsymbol{I}-\frac{\boldsymbol{b} \boldsymbol{v}^{\prime}}{\boldsymbol{b} \cdot \boldsymbol{v}}\right)
$$

is the covariance matrix with rank $n-1$.

For the application in Sections 3-5, we consider the special case in which $\boldsymbol{A}$ is a diagonalizable matrix. Then, there exist column vectors $\hat{u}_{i}$ and $\hat{v}_{i}$, such that, $\hat{u}_{i}^{\prime} \boldsymbol{A}=\gamma_{i} \hat{u}_{i}^{\prime}, \boldsymbol{A} \hat{v}_{i}=\gamma_{i} \hat{v}_{i}$, and $\hat{u}_{i} \cdot \hat{v}_{j}=\delta_{i j}$ for $i, j=1, \ldots, n$. Using Lemma 5.3 in [8], the matrices $\boldsymbol{P}$ and $\boldsymbol{P} e^{s \boldsymbol{A}}$ can now be expressed as

$$
\boldsymbol{P}=\sum_{j: \gamma_{j} \in \Delta_{1}} \hat{v}_{j} \hat{u}_{j}^{\prime} \quad \text { and } \quad \boldsymbol{P} e^{s \boldsymbol{A}}=\sum_{j: \gamma_{j} \in \Delta_{1}} e^{s \gamma_{j}} \hat{v}_{j} \hat{u}_{j}^{\prime},
$$

respectively, and hence $\boldsymbol{\Sigma}_{\boldsymbol{I}}$ becomes

$$
\boldsymbol{\Sigma}_{\boldsymbol{I}}=\sum_{j: \gamma_{j} \in \Delta_{1}} \sum_{k: \gamma_{k} \in \Delta_{1}} \frac{\hat{u}_{j}^{\prime} \boldsymbol{B} \hat{u}_{k}}{\gamma-\gamma_{j}-\gamma_{k}} \hat{v}_{j} \hat{v}_{k}^{\prime}
$$

Author Contributions: Development of mathematical models and analytical calculations, IK, DT and KB; provision of biological data, MM, PP and SS; implementation of statistical software, KB and DT; data analysis, manuscript writing and editing, DT.

Funding: KB was supported by Vetenskapsrådets grant no. 2017-04951. MM and PP were partially supported by IMB PAS.

Conflicts of Interest: The authors declare no conflict of interest. The funders had no role in the design of the study; in the collection, analyses, or interpretation of data; in the writing of the manuscript, or in the decision to publish the results.

\section{Abbreviations}


The following abbreviations are used in this article:

BiSSE binary state speciation and extinction

MuSSE multistate speciation and extinction

cnf1 cytotoxic necrotizing factor

fyuA pesticin receptor protein

iroN IroN protein

papC fimbrial protein

VF virulence factor

ast A heat-stable enterotoxin

fimG fimbrial protein

hly1 alpha hemolysin

iutA ferric aerobactin receptor

sat secreted autotransporter toxin

\section{REFERENCES}

[1] K. Arbuckle and M. P. Speed, Antipredator defenses predict diversification rates, PNAS, 112, 13597-13602 (2015). doi: 10.1073/pnas.1509811112. Cited on p. 60.

[2] K. B. Athreya, Some results on multitype continuous time Markov branching processes, Ann. Math. Stat. 39, 347-357 (1968). doi: 10.1214/aoms/1177698395; MR 0221600 Cited on pp. 61, 62, and 82.

[3] K. B. Athreya and P. E. Ney, Branching Processes, Dover Publications Inc. New York, 1972. Cited on pp. 61 and 62.

[4] K. Bartoszek, M. Majchrzak, S. Sakowski, A. B. Kubiak-Szeligowska, I. Kaj, P. Parniewski, Predicting pathogenicity behavior in Escherichia coli population through a state dependent model and TRS profiling, PLOS Comput. Biol. 14, e1005931 (2018). doi: 10.1371/journal.pcbi.1005931; PubMed Central PMCID: PMC5809097 . Cited on pp. 59, 60, 66, 67, 73,80 , and 81.

[5] S. L. Chen, M. Wu, J. P. Henderson, T. M. Hooton, M. E. Hibbing, S. J. Hultgren, J. I. Gordon, Genomic diversity and fitness of E. coli strains recovered from the intestinal and urinary tracts of women with recurrent urinary tract infection, Sci. Transl. Med. 5, 184ra60 (2013). doi: 10.1126/scitranslmed.3005497. Cited on p. 67.

[6] A. S. Cross, What is a virulence factor? Crit. Care. 12, 196 (2008). doi: 10.1186/cc7127. Cited on p. 60.

[7] R. G. FitzJohn, Diversitree: comparative phylogenetic analyses of diversification in R, Methods Ecol. Evol. 3, 1084-1092 (2012). doi: 10.1111/j.2041-210X.2012.00234.x. Cited on pp. 60, 67, 68, and 75.

[8] S. Janson, Functional limit theorems for multitype branching processes and generalized Pólya urns, Stoch. Proc. Appl. 110, 177-245 (2004). doi: 10.1016/j.spa.2003.12.002. Cited on pp. 61, 62, 71, 81, 82, and 83.

[9] R. A. Johnson and D. W. Wichern, Applied multivariate statistical analysis, Pearson Education Inc. New Jersey, 2007. Cited on p. 71.

[10] I. Jorgensen, P. C. Seed, How to Make It in the Urinary Tract: A Tutorial by Escherichia coli, PLoS Pathog. 8, e1002907 (2012). doi: 10.1371/journal.ppat.1002907. Cited on p. 67.

[11] S. Kitamoto, H. Nagao-Kitamoto, P. Kuffa, N. Kamada, Regulation of 
virulence: the rise and fall of gastrointestinal pathogens, J. Gastroenterol. 51, 195-205 (2016). doi: 10.1007/s00535-015-1141-5. Cited on p. 81.

[12] W. P. Maddison, P. E. Midford, S. P. Otto, Estimating a Binary Character's Effect on Speciation and Extinction, Syst. Biol. 56, 701-710 (2007). doi: 10.1080/10635150701607033. Cited on p. 59.

[13] Maple 18.00 (2014). Maplesoft, a division of Waterloo Maple Inc., Waterloo, Ontario. Cited on pp. 64 and 74.

[14] M. D. Pirie, E. G. H. Oliver, A. Mugrabi de Kuppler, B. Gehrke, N. C. Le Maitre, M. Kandziora, D. U. Bellstedt, The biodiversity hotspot as evolutionary hot-bed: spectacular radiation of Erica in the Cape Floristic Region, BMC Evol. Biol. 16, 190 (2016). doi: 10.1186/s12862-016-0764-3. Cited on p. 60.

[15] R Core Team, R: A language and environment for statistical computing, R Foundation for Statistical Computing, Vienna, Austria, URL https://www.R-project.org/, 2016. Cited on p. 67.

[16] J. L. Sachs, R. G. Skophammer, N. Bansal, J. E. Stajich, Evolutionary origins and diversification of proteobacterial mutualists, Proc. R. Soc. B 281: 20132146 (2013). doi: 10.1098/rspb.2013.2146. Cited on p. 60.

[17] G. E. Schwarz, Estimating the dimension of a model, Ann. Stat. 6, 461454 (1978). doi: 10.1214/aos/1176344136. Cited on p. 68.

\title{
Zastosowanie wielorodzajowego procesu gałązkowego do analizy patogenności bakterii
}

\author{
Daniah Tahir, Ingemar Kaj, Krzysztof Bartoszek, \\ Marta Majchrzak, Paweł Parniewski, Sebastian Sakowski
}

\begin{abstract}
Streszczenie W celu zbadania patogenności szczepów E. coli, bakterii z rodzaju Escherichia, użyto wielorodzajowego markowskiego procesu gałązkowego z czasem ciągłym. W pierwszej kolejności zrobiono przegląd własności wykorzystywanego procesu wraz z najważniejszymi wynikami granicznymi opisującymi zachowanie procesu przy różnych założeniach. Następnie przyjęto konkretny model, w którym rozgałęzenia są zależne od stanu oraz zastosowano go do badania patogenności w zestawie 251 szczepów E. coli pochodzących z II Centralnego Szpitala Klinicznego Wojskowej Akademii Medycznej. Parametry modelu, tempa narodzin oraz mutacji, zostały uzyskane metodą największej wiarygodności. Przedstawiona w artykule analiza potwierdza znane własności patogenności bakterii oraz sugeruje nowe ścieżki pracy badawczej.
\end{abstract}

2010 Klasyfikacja tematyczna AMS (2010): 60B12; $60 \mathrm{~J} 85$.

Stowa kluczowe: czynniki patogenności, model markowski, proces gałązkowy, szczepy E. coli, twierdzenia graniczne. 


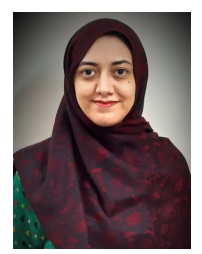

Daniah Tahir obtained her $\mathrm{PhD}$ in Applied Mathematics and Statistics from Uppsala University, Sweden, in 2019 and MPhil in Mathematics from National University of Sciences and Technology, Pakistan, in 2013. Her research interests include stochastic processes in evolutionary biology, phylogenetics, and epidemiological modeling.

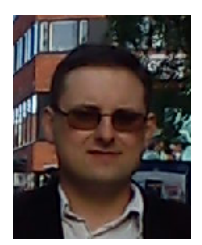

Krzysztof Bartoszek is currently a lecturer of Statistics at the University of Linköping. He is a Computational Biology graduate from the University of Cambridge (MPhil), and has a doctorate in Mathematical Statistics, obtained in 2013 at the University of Gothenburg. His main interests are associated with stochastic processes in phylogenetics.

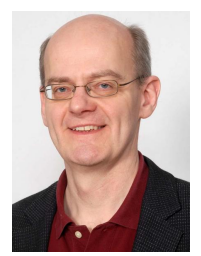

Ingemar Kaj is a Professor in Mathematical Statistics at Uppsala University since 1992 with research interests in stochastic processes, random fields, long range dependence, and stochastic models in evolutionary biology and other application areas.

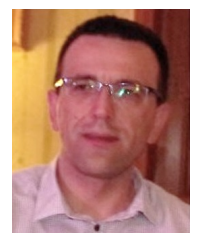

Sebastian Sakowski received his $\mathrm{PhD}$ degree in Computer Science from Silesian University of Technology in 2011, and MSc in Computer Science from University of Lodz in 2004. He is a member of Polish Bioinformatics Society. His current interests include automata theory, DNA computing, bioinformatics and computational biology.

Marta Majchrzak, Ph.D., is a molecular biologist at the Laboratory of Molecular Genetics, Institute of Medical Biology, Polish Academy of Sciences, Poland. Her research interest are in biochemistry and microbiology.

Pawel Parniewski, Ph.D., is the Head of the Laboratory of Molecular Genetics at the Institute of Medical Biology, Polish Academy of Sciences, Poland. His research interests are in microbiology and molecular biology.

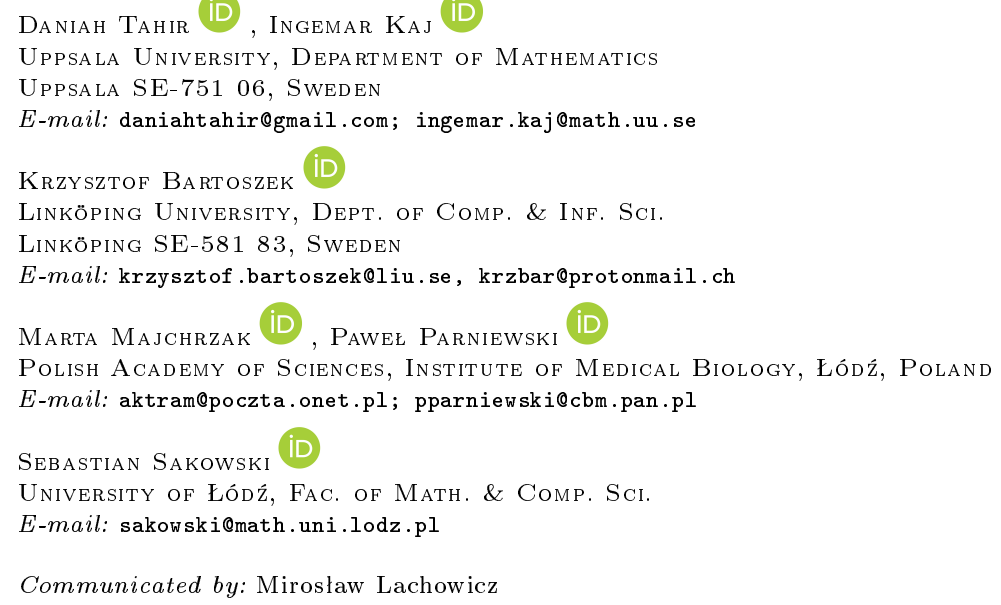

